\title{
UNIVERSITÄT KAISERSLAUTERN
}

\section{DEFORMATIONS OF MAXIMAL COHEN-MACAULAY MODULES}

Gerhard Pfister and Dorin Popescu

Preprint Nr. 257

ISSN 0943-8874

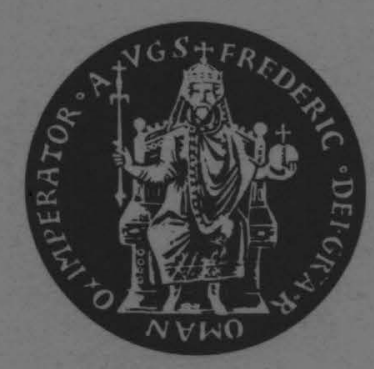

FACHBEREICH MATHEMATIK 


\title{
Deformations of maximal Cohen-Macaulay modules
}

\author{
Gerhard Pfister \\ Dorin Popescu \\ Universität Kaiserslautern \\ Institute of Mathematics \\ Fachbereich Mathematik \\ University of Bucharest \\ Postfach 3049 \\ P.O. Box 1-764 \\ 67653 Kaiserslautern \\ Germany \\ Bucharest 70700 \\ Romania
}

October 10, 1994

\section{Contents}

Introduction

1 Reduction ideals for deformations $\quad 3$

2 Matrix factorizations for liftings $\quad 9$

3 Knörrer's Periodicity Theorem for liftings 14

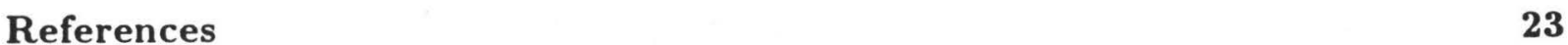




\section{Introduction}

Let $(R, m)$ be a complete Cohen-Macaulay isolated singularity over a field $K$ which is either perfect or $\left[K: K^{p}\right]<\infty$ if $p:=$ char $K>0$. According to Dieterich [Di] and [Yo] Ch. 6 (see also [Pol], [PR] or, more generally, [CHP]) there exists a system of parameters $x=\left(x_{1}, \ldots, x_{r}\right)$ of $R$ such that the base change functor $R /(x) \otimes_{R}$ - defines an injection $\nu$ preserving the indecomposability from the set of isomorphism classes of maximal CohenMacaulay $R$-modules to the set of isomorphism classes of $R /(x)$-modules (in this case the ideal generated by $x$ is called (after [Di]) a reduction ideal. Trying to describe the image of $\nu$ we noticed in [Po2] that a finitely generated $R /(x)$-module is in $\operatorname{Im} \nu$ if and only if it has the form $x_{1} \ldots x_{r} P$ for a finitely generated $R_{2}:=R /\left(x_{1}^{2}, \ldots, x_{r}^{2}\right)$-module satisfying

$$
\left(\left(x_{1}, \ldots, x_{j-1}\right) P: x_{j}\right)_{P}=\left(x_{1}, \ldots, x_{j}\right) P
$$

for all $j, 1 \leq j \leq r$.

In Section 1 we extend these results in the frame of deformations of maximal CohenMacaulay $R$-modules. Let $A$ be a Noetherian, Henselian local $K$-algebra with residue field $K$. Then the base change functor defines a bijection preserving the indecomposability from the set of isomorphism classes of modules which deform maximal Cohen-Macaulay $\mathcal{R}$-modules over $A$ onto the set of isomorphism classes of modules which deform over $A$ the $R /(x)$-module of the form $x_{1} \ldots x_{r} P$ for a finitely generated $R_{2}$-module $P$ satisfying $(\mathcal{L})$ as above (see Theorem 1.4). Unfortunately, the isomorphisms considered here are only the isomorphisms as modules and not as deformations (see 1.2). In fact, given $M \in \mathrm{MCM}(R)$ the base change functor induces a non-bijective map from the set of isomorphism classes of deformations of $M$ over $A$ to the set of isomorphism classes of deformations of $M /(x) M$ over $A$ (see Example 1.18). As a main tool in proving Theorem 1.4 (and in the whole paper as well) we used the so-called Auslander-Ding-Solberg lifting theory (see [ADS]).

In Section 2 we extended Eisenbud's Matrix Factorization Theorem (see [Ei] or [Yo] Ch. 7) in the frame of deformations of maximal Cohen-Macaulay modules over hypersurfaces. Let $K$ be a field, $(B, \wp, K)$ a Noetherian local ring, $G \in B[[X]], X=\left(X_{1}, \ldots, X_{r}\right)$ a non-unit formal power series, $\mathcal{R}:=B[[X]] /(G)$ and $R:=K \otimes_{B} \mathcal{R}$. Suppose $\mathcal{R}$ is flat over $B$. Then the category $\mathcal{D}_{B}^{R}$ of $R$-modules, which deform maximal Cohen-Macaulay $R$-modules over $B$, is equivalent to the category of matrix factorizations of $G$ over $B[[X]]$ modulo $\{(1, G)\}$ (see Theorem 2.8). One of the main applications of Eisenbud's Matrix Factorization Theorem was Knörrer's Periodicity Theorem, which was very useful in the theory of simple singularities (see $[\mathrm{Kn}],[\mathrm{BGS}],[\mathrm{Sc}],[\mathrm{GKr}])$. We were surprised to see that in characteristic two the result was not completely established (Solberg gave a proof in [So] only for hypersurfaces of finite Cohen-Macaulay type). It is the purpose of our Section 3 to fulfill this small gap and to extend the result in the frame of deformations of maximal Cohen-Macaulay modules over hypersurfaces. Let $U, V$ be some new indeterminates, $\mathcal{R}^{\prime}:=B[[X, U, V]] /(G+U V)$ and $\mathcal{R}^{\prime}:=K \otimes_{B} \mathcal{R}^{\prime}$. Suppose $B$ is Henselian and $\mathcal{R}^{\prime}$ is flat over $B$. Then the category $\mathcal{D}_{B}^{R}$ is stable equivalent to the category $\mathcal{D}_{B}^{R^{\prime}}$ of all $\mathcal{R}^{\prime}$-modules which deform maximal Cohen-Macaulay $R^{\prime}$-modules over $B$ (see 3.17 ), that is, there exists an equivalence

$$
F: \mathcal{D}_{B}^{R} /\{\mathcal{R}\} \rightarrow \mathcal{D}_{B}^{R^{\prime}} /\left\{\mathcal{R}^{\prime}\right\}
$$


(for notations cf. [Yo]).

Let $M$ be an indecomposable maximal Cohen-Macaulay $R$-module. Then there exists a bijection from the set of isomorphism classes of deformations of $M$ over $B$ onto the set of isomorphism classes of deformations of $F(M)$ over $B$ (see 3.20).

The main part of this paper was completed while the second author visited the University of Kaiserslautern in the frame of a grant from the Deutsche Forschungsgemeinschaft (DFG). Some preparations were done by him in the Romanian Institute of Mathematics, University of Bucharest and whilst he visited the Sonderforschungsbereich 170 in Göttingen. We are grateful to all these institutions for support and hospitality and we owe thanks to R.-O. Buchweitz, H. Flenner, G.-M. Greuel and M. Roczen for some very helpful discussions. 


\section{Reduction ideals for deformations}

1.1 Let $(R, m)$ be a complete Cohen-Macaulay local ring containing its residue field $K$. We suppose $R$ is an isolated singularity and $K$ is perfect, or $\left[K: K^{p}\right]<\infty$ if $p:=$ char $K>0$. Let $\operatorname{MCM}(R)$ be the category of maximal Cohen-Macaulay $R$-modules and $\mathcal{C}_{K}$ the category of Noetherian, Henselian local $K$-algebras with residue fields $K$.

1.2 Given $A \in \mathcal{C}_{K}$, let $R_{A}:=R \widehat{\otimes}_{K} A$ be the completion of $R \otimes_{K} A$ with respect to $m \otimes_{K} A$. Then $R_{A}$ is a Noetherian local flat $A$-algebra (see [GP] Appendix). A deformation of a finitely generated $R$-module $N$ over $A$ is a finitely generated $R_{A}$-module $L$, which is flat over $A$, together with an isomorphism $N \cong L \otimes_{A} K$. Two deformations $L$ and $L^{\prime}$ of $N$ over $A$ are isomorphic if an isomorphism $\phi: L \rightarrow L^{\prime}$ of $R_{A}$-modules exists such that the following diagram commutes

$$
\begin{array}{ccc}
N & \stackrel{\sim}{\rightarrow} & L \otimes_{A} K \\
\| & & \downarrow \\
N & \stackrel{\sim}{\rightarrow} & L^{\prime} \otimes_{A} K
\end{array}
$$

where the second vertical map is induced by $\phi$. $\operatorname{Def}_{N}(A)$ denotes the set of isomorphism classes of deformations of $N$ over $A$.

1.3 Let $A \in \mathcal{C}_{K}$ and $\mathcal{D}_{A}$ be the category of all finitely generated $R_{A}$-modules $E$ which are flat over $A$ and such that $K \otimes_{A} E \in \operatorname{MCM}(R)$. Roughly speaking, $\mathcal{D}_{A}$ is the category of all deformations of modules of $\operatorname{MCM}(R)$ over $A$. Using [BH] (1.2.17) in the case $A \rightarrow R_{A}$ we see that a system of parameters $y$ of $R$ is regular for every module $E \in \mathcal{D}_{A}$ and $E / y E$ is still flat over $A$.

Theorem 1.4 There exists a system of parameters $x=\left(x_{1}, \ldots, x_{r}\right)$ of $R$ such that the base change functor $\left(R_{1}\right)_{A} \otimes_{R_{A}}-: \mathcal{D}_{A} \rightarrow \operatorname{Mod}\left(R_{1}\right)_{A}, R_{1}:=R /(x)$ defines a bijection preserving the indecomposability from the set of isomorphism classes of modules of $\mathcal{D}_{A}$ onto the set of isomorphism classes of those $\left(R_{1}\right)_{A}$-modules which deform $R_{1}$-modules of type $x_{1} \ldots x_{r} P$, where $P$ is a finitely generated $R_{2}:=R /\left(x_{1}^{2}, \ldots, x_{r}^{2}\right)$-module satisfying

$$
\left(\left(x_{1}, \ldots, x_{j-1}\right) P: x_{j}\right)_{P}=\left(x_{1}, \ldots, x_{j}\right) P \text { for all } j, 1 \leq j \leq r .
$$

For the proof we need some preparations.

1.5 Let $y=\left(y_{1}, \ldots, y_{r}\right)$ be a system of parameters of $R$, so an $R$-sequence. Then $C_{y}:=$ $K[[y]]$ is a regular local subring of $R$ and $R$ is finite and free over $C_{y}$. The Noether different $\mathcal{N}_{R / C_{y}}$ is defined by $\mathcal{N}_{R / C_{y}}:=\rho\left(((0): \operatorname{Ker} \rho)_{R \otimes_{C_{y}} R}\right)$, where $\rho: R \otimes_{C_{y}} R \rightarrow R$ is given by $u \otimes u^{\prime} \rightarrow u u^{\prime}$. Let $\mathcal{N}_{R}:=\sum_{y} \mathcal{N}_{R / C_{y}}$, where the sum is made over all systems of parameters $y$ of $R . \mathcal{N}_{R}$ is an $m$-primary ideal of $R$, since it defines the singular locus of $R$ (see [Yo] (6.12), or [Pol] (2.10)).

Lemma $1.6 \mathcal{N}_{R_{A} /\left(C_{y}\right)_{A}}=\mathcal{N}_{R / C_{y}} R_{A}$.

Proof: We have $\left(C_{y}\right)_{A} \otimes_{C_{y}}\left(R \otimes_{C_{y}} R\right) \cong\left(\left(C_{y}\right)_{A} \otimes_{C_{y}} R\right) \otimes_{C_{y}}\left(\left(C_{y}\right)_{A} \otimes_{C_{y}} R\right) \cong R_{A} \otimes_{\left(C_{y}\right)_{A}} R_{A}$ since $R$ is finite over $C_{y}$. By flatness Ker $\rho$ generates in $R_{A} \otimes_{\left(C_{y}\right)_{A}} R_{A}$ the kernel of the map $\rho_{A}: R_{A} \otimes\left(C_{y}\right)_{A} R_{A} \rightarrow R_{A}$ defined by $\rho$. Again using the flatness of $\left(C_{y}\right)_{A}$ over $C_{y}$ we note that $((0): \operatorname{Ker} \rho)_{R \otimes_{C_{y}} R}$ generates $\left((0): \operatorname{Ker} \rho_{A}\right)_{R_{A} \otimes_{\left(C_{y}\right)_{A}} R_{A}}$ which is enough. 
Proposition 1.7 $\mathcal{N}_{R} \operatorname{Ext}_{R_{A}}^{1}(E, F)=0$ holds for all $E \in \mathcal{D}_{A}$ and all finitely generated $R_{A^{-}}$ modules $F$.

Proof: Fix $E, F$. It is enough to show that

$$
\mathcal{N}_{R / C_{y}} \operatorname{Ext}_{R_{A}}^{1}(E, F)=0
$$

for an arbitrary system of parameters $y$ of $R$. Note that $K \otimes_{A} E \in \operatorname{MCM}(R)$ is free over $C_{y}$ since $C_{y}$ is regular. Then $E$ as a $\left(C_{y}\right)_{A}$-module is a deformation of a free $C_{y}$-module and so $E$ is free over $\left(C_{y}\right)_{A}$. Now the idea of our proof is as in [PR] (1.4) (see also [Di], Lemma (1.5)), although $\left(C_{y}\right)_{A}$ is not a regular ring (important here is just the fact that our modules from $\mathcal{D}_{A}$ are free by restriction to $\left.\left(C_{y}\right)_{A}\right)$. Indeed, let $G$ be the first syzygy of $E$ over $R_{A}$, that is, we have the following exact sequence

$$
0 \rightarrow G \rightarrow R_{A}^{s} \rightarrow E \rightarrow 0
$$

for a certain $s \in \mathbb{N}$. Since $E$ is free over $\left(C_{y}\right)_{A}$ we obtain the following exact sequence of $R_{A}$-bimodules

$$
0 \rightarrow \operatorname{Hom}_{\left(C_{y}\right)_{A}}(E, F) \rightarrow \operatorname{Hom}_{\left(C_{y}\right)_{A}}\left(R_{A}^{s}, F\right) \rightarrow \operatorname{Hom}_{\left(C_{y}\right)_{A}}(G, F) \rightarrow 0 .
$$

Applying the Hochschild cohomology functors (see [Pi] Ch. 11 for details) we obtain the exact sequence

$$
\begin{gathered}
0 \rightarrow H_{\left(C_{y}\right)_{A}}^{0}\left(R_{A}, \operatorname{Hom}_{\left(C_{y}\right)_{A}}(E, F)\right)=\operatorname{Hom}_{R_{A}}(E, F) \rightarrow \operatorname{Hom}_{R_{A}}\left(R_{A}^{s}, F\right) \rightarrow \\
\operatorname{Hom}_{R_{A}}(G, F) \rightarrow H_{\left(C_{y}\right)_{A}}^{1}\left(R_{A}, \operatorname{Hom}_{\left(C_{y}\right)_{A}}(E, F)\right) .
\end{gathered}
$$

Clearly the image of the last map above is $\operatorname{Ext}_{R_{A}}^{1}(E, F)$ and so it is enough to show that

$$
\mathcal{N}_{R / C_{y}} H_{\left(C_{y}\right)_{A}}^{1}\left(R_{A}, \operatorname{Hom}_{\left(C_{y}\right)_{A}}(E, F)\right)=0
$$

As $H_{\left(C_{y}\right)_{A}}^{1}\left(R_{A}, \operatorname{Hom}_{\left(C_{y}\right)_{A}}(E, F)\right)$ is a quotient of

$$
\operatorname{Der}_{\left(C_{y}\right)_{A}}^{1}\left(R_{A}, \operatorname{Hom}_{\left(C_{y}\right)_{A}}(E, F)\right)
$$

we obtain (1), since $\mathcal{N}_{R / C_{y}} \Omega_{R_{A} /\left(C_{y}\right)_{A}}=0$ (see [Yo] Ch. 6, or [Pol] $(2.10)$ ), $\Omega_{R_{A} /\left(C_{y}\right)_{A}}$ being the differential module of $R_{A}$ over $\left(C_{y}\right)_{A}$.

Proposition 1.8 Let $z=\left(z_{1}, \ldots, z_{r}\right)$ be a system of parameters of $R, e_{1}, \ldots, e_{r}$ some positive integers such that $z_{i}^{e_{i}} \in \mathcal{N}_{R}$ for $1 \leq i \leq r, x_{i}:=z_{i}^{e_{i}+1}$ and $x=\left(x_{1}, \ldots, x_{r}\right)$. Then the base change functor $\left(R_{1}\right)_{A} \otimes_{R_{A}}-: \mathcal{D}_{A} \rightarrow \operatorname{Mod}\left(R_{1}\right)_{A}, R_{1}:=R /(x)$ defines an injection from the isomorphism classes of indecomposable modules of $\mathcal{D}_{A}$ to the isomorphism classes of indecomposable $\left(R_{1}\right)_{A}$-modules.

Proof: Since $z_{i}^{e_{i}} \operatorname{Ext}_{R_{A}}^{1}(E, F)=0$ for all $E \in \mathcal{D}_{A}$ and each finitely generated $R_{A}$-module $F$ (see 1.7), we can show similarly as in [PR] (2.1), (2.2) that given $E \in \mathcal{D}_{A}, F$ a finitely generated $R_{A}$-module, $\tilde{R}:=R /\left(z_{1}^{e_{1}}, \ldots, z_{r}^{e_{r}}\right)$ and a linear $R_{A}$-map $\phi:\left(R_{1}\right)_{A} \otimes_{R_{A}} E \rightarrow$ $\left(R_{1}\right)_{A} \otimes_{R_{A}} F$ there exists a linear $R_{A}-$ map $\psi: E \rightarrow F$ such that $\tilde{R}_{A} \otimes_{\left(R_{1}\right)_{A}} \phi \cong \tilde{R}_{A} \otimes_{R_{A}} \psi$. This is enough using a variant of [Yo] $(6.16),(6.18)$ (here we need that $R_{A}$ and so $A$ are Henselian!). 
Remark 1.9 The proofs of 1.7, 1.8 are entirely based on Yoshino's ideas, the only difference is that we prefer to work with $\operatorname{Ext}_{R}^{1}(-,-)$ as in [Di], [PR], [CHP] (3.10), (3.11) instead of the first Hochschild cohomology functor.

Applying Proposition 1.8 we find the system $x$ which satisfies Theorem 1.4 (Proposition 1.8 is an analog of Dieterich-Yoshino's result in the frame of deformations of maximal CohenMacaulay modules). To show that $x$ really satisfies 1.4 means to extend [Po2], Section 1, in the frame of deformations. For this aim we need the concept of lifting in the sense of Auslander-Ding-Solberg [ADS] (see also [DS]) which we will recall below.

Definition 1.10 Let $\psi: \Lambda \rightarrow \Gamma$ be a morphism of Noetherian rings and $M$ a finitely generated $\Gamma$-module. A finitely generated $\Lambda$-module $L$ is a lifting of $M$ to $\Lambda$ (see [ADS]) if $M \cong \Gamma \otimes_{\Lambda} L$ and $\operatorname{Tor}_{i}^{\wedge}(L, \Gamma)=0$ for all $i \geq 1$.

Let Lift $(\Lambda, \Gamma)$ be the set of all finitely generated $\Lambda$-modules $L$ such that $\operatorname{Tor}_{i}^{\Lambda}(\Gamma, L)=0$ for each $i \geq 1$ and let

$$
\operatorname{lift}(\Lambda, \Gamma)=\left\{\Gamma \otimes_{\Lambda} L \mid L \in \operatorname{Lift}(\Lambda, \Gamma)\right\} .
$$

Notice that $\operatorname{Lift}\left(R_{A}, R\right)$ is the set of deformations of all finitely generated $R$-modules over $A$.

Indeed, we have $\operatorname{Tor}_{i}^{A}(K, E) \cong \operatorname{Tor}_{i}^{R_{A}}(R, E)$ for all $i \geq 1$ and all $R_{A}$-modules $E$. Thus, $E \in \operatorname{Lift}\left(R_{A}, R\right)$ if and only if $\operatorname{Tor}_{i}^{A}(K, E)=0, i \geq 1$, that is, if and only if $E$ is flat over $A$ by the local flatness criterium (see [Ma] (20.C)).

Remark 1.11 i) Let $\Lambda \rightarrow \Gamma \rightarrow \Delta$ be two ring morphisms and $L$ a finitely generated $\Lambda$-module. If $L \in \operatorname{Lift}(\Lambda, \Gamma)$ then $L \in \operatorname{Lift}(\Lambda, \Delta)$ if and only if $\Gamma \otimes_{\Lambda} L \in \operatorname{Lift}(\Gamma, \Delta)$ $($ see $[\mathrm{Po} 2](1.7))$.

ii) Let $y$ be a regular sequence of elements in $\Gamma$ and suppose that $\Delta=\Gamma /(y)$. Then $\operatorname{Lift}(\Gamma, \Delta)$ is the set of all $\Gamma$-modules for which $y$ is a regular system. Moreover, $L \in \operatorname{Lift}(\Lambda, \Delta)$ if and only if $L \in \operatorname{Lift}(\Lambda, \Gamma)$ and $\Gamma \otimes_{\Lambda} L \in \operatorname{Lift}(\Gamma, \Delta)$ (see, for example, [Po2] (1.15)).

iii) Let $z=\left(z_{1}, \ldots, z_{r}\right)$ be a system of elements in $\Gamma$ satisfying $\left(\left(z_{1}, \ldots, z_{j-1}\right): z_{j}\right)=$ $\left(z_{1}, \ldots, z_{j}\right)$ for all $j, 1 \leq j \leq r$ and suppose that $\Delta=\Gamma /(z)$. Then $L \in \operatorname{Lift}(\Lambda, \Delta)$ if and only if $L \in \operatorname{Lift}(\Lambda, \Gamma)$ and $\Gamma \otimes_{\Lambda} L \in \operatorname{Lift}(\Gamma, \Delta)$ (see [Po2] (1.12)). Moreover, if $E$ is a finitely generated $\Gamma$-module then $E \in \operatorname{Lift}(\Gamma, \Delta)$ if and only if $\left(\left(z_{1}, \ldots, z_{j-1}\right) E: z_{j}\right)_{E}=\left(z_{1}, \ldots, z_{j}\right) E$ for all $j, 1 \leq j \leq r$ (see [Po2] (1.11)).

iv) $\mathcal{D}_{A}=\operatorname{Lift}\left(R_{A}, R /(y)\right)$ holds for any system of parameters $y$ of $R$. Indeed, $y$ is an $R$-sequence and by ii) we have $E \in \operatorname{Lift}\left(R_{A}, R /(y)\right)$ if and only if $E \in \operatorname{Lift}\left(R_{A}, R\right)$ and $K \otimes_{A} E \cong R \otimes_{R_{A}} E \in \operatorname{Lift}(R, R /(y))$, that is, if and only if $E$ is flat over $A$ (see $1.10)$ and $y$ is a $K \otimes_{A} E$-sequence (see ii)).

v) A finitely generated $R_{A}$-module $E$ belongs to $\mathcal{D}_{A}$ if and only if a system of parameters $y$ of $R$ (and then any) is regular on $E$ and $E / y E$ is flat over $A$. Indeed, it is enough to show that $E \in \operatorname{Lift}\left(R_{A}, R /(y)\right)$ if and only if $E \in \operatorname{Lift}\left(R_{A}, R_{A} /(y)\right)$ and $E / y E \in$ $\operatorname{Lift}\left(R_{A} /(y), R /(y)\right)$ by ii) and 1.10. The sufficiency follows from i). For the necessity see that $E \in \operatorname{Lift}\left(R_{A}, R /(y)\right)$ since $y$ is regular on $E$ by 1.3. Then apply i). 
Lemma 1.12 Let $S \in \mathcal{C}_{K}$ be a complete local ring, $z=\left(z_{1}, \ldots, z_{r}\right)$ a system of elements of $S$ such that $\left(\left(z_{1}, \ldots, z_{j-1}\right): z_{j}\right)=\left(z_{1}, \ldots, z_{j}\right)$ and $z_{j}^{2}=0$ for all $j, 1 \leq j \leq r, S_{1}:=S /(z)$, $A \in \mathcal{C}_{K}, S_{A}:=S \widehat{\otimes}_{K} A$ and $E$ a finitely generated $S_{A}$-module. The following statements are equivalent:

i) $E \in \operatorname{Lift}\left(S_{A}, S_{1}\right)$,

ii) $E$ is flat over $A$ and $N:=K \otimes_{A} E$ satisfies $\left(\left(z_{1}, \ldots, z_{j-1}\right) N: z_{j}\right)_{N}=\left(z_{1}, \ldots, z_{j}\right) N$ for all $j, 1 \leq j \leq r$,

iii) $E$ satisfies $\left(\left(z_{1}, \ldots, z_{j-1}\right) E: z_{j}\right)_{E}=\left(z_{1}, \ldots, z_{j}\right) E$ for all $j, 1 \leq j \leq r$ and $E /(z) E$ is a flat $A$-module.

Proof: By 1.11 iii) applied to $S_{A} \rightarrow S \rightarrow S_{1}$ we obtain $E \in \operatorname{Lift}\left(S_{A}, S_{1}\right)$ if and only if $E \in$ Lift $\left(S_{A}, S\right)$ (that is, $E$ is flat over $A$ by 1.10) and $S \otimes_{S_{A}} E \cong K \otimes_{A} E=N \in \operatorname{Lift}\left(S, S_{1}\right)$ (that is, $N$ satisfies the property from ii)). Thus i) $\Leftrightarrow$ ii). Now iii) states that $E \in \operatorname{Lift}\left(S_{A},\left(S_{1}\right)_{A}\right)$ and $E /(z) E \in \operatorname{Lift}\left(\left(S_{1}\right)_{A}, S_{1}\right)$. Thus iii) $\Rightarrow$ i) by 1.11 i).

It remains to prove the difficult part, namely ii) $\Rightarrow$ iii). Apply induction on $r$. Let $r=1$. Tensorizing with $K \otimes_{A}$ - the exact sequence

$$
0 \rightarrow(0: z)_{E} \rightarrow E \stackrel{z}{\rightarrow} z E \rightarrow 0,
$$

we obtain the following exact sequence

$$
0=\operatorname{Tor}_{1}^{A}(K, E) \rightarrow \operatorname{Tor}_{1}^{A}(K, z E) \rightarrow K \otimes_{A}(0: z)_{E} \stackrel{\gamma}{\rightarrow} N \rightarrow K \otimes_{A} z E \rightarrow 0,
$$

where $\operatorname{Im} \gamma \subset(0: z)_{N}=z N$. Since $z^{2}=0$ we have $(0: z)_{E} \supset z E$ and $\operatorname{Im} \gamma \supset z N$ follows. Thus $\operatorname{Im} \gamma=z N$. Then $K \otimes_{A} z E \cong N / z N \cong z N$ because the sequence

$$
0 \rightarrow z N \rightarrow N \stackrel{z}{\rightarrow} z N \rightarrow 0
$$

is exact by ii).

Tensorizing by $K \otimes_{A}-$ the exact sequence

$$
0 \rightarrow z E \rightarrow E \rightarrow E / z E \rightarrow 0
$$

we obtain the following exact sequence

$$
0=\operatorname{Tor}_{1}^{A}(K, E) \rightarrow \operatorname{Tor}_{1}^{A}(K, E / z E) \rightarrow K \otimes_{A} z E \stackrel{\tau}{\rightarrow} N \rightarrow K \otimes_{A} E / z E \rightarrow 0,
$$

where the image of $\tau$ is exactly $z N$. Thus, $K \otimes_{A}(E / z E) \cong N / z N \cong z N$ and $\tau$ defines a surjection $z N \cong K \otimes_{A} z E \stackrel{\tau^{\prime}}{\rightarrow} z N$, which is also an injection. Hence, $\operatorname{Tor}_{1}^{A}(K, E / z E)=0$. By the local flatness criterium (see [Ma] (20.C)) we obtain that $E / z E$ is flat over $A$. Using (5), $z E$ flat over $A$ follows, too, and so $\gamma$ from (4) is an injection.

Tensorizing the inclusions $z E \rightarrow E,(0: z)_{E} \rightarrow E$ by $K \otimes_{A}-$ we obtain the isomorphisms $K \otimes_{A} z E \stackrel{\sim}{\rightarrow} z N$ and $K \otimes_{A}(0: z)_{E} \stackrel{\sim}{\rightarrow} z N$ given by $\gamma$ and $\tau^{\prime}$. Thus, the inclusion $u: z E \rightarrow$ $(0: z)_{E}$ induces via $K \otimes_{A}-$ a bijection and $S \otimes_{S_{A}}$ Coker $u \cong K \otimes_{A}$ Coker $u=0$ follows. By Nakayama's Lemma we obtain Coker $u=0$, that is, $(0: z)_{E}=z E$. If $r>1$ then, as above, $E^{\prime}:=E / z_{1} E$ is flat over $A$ and by induction hypothesis we are finished. $\mathcal{D}_{A}$.

The following Proposition is an extension of [Po2] (1.16) in the frame of modules from 
Proposition 1.13 Let $S \in \mathcal{C}_{K}$ be a complete local ring, $y=\left(y_{1}, \ldots, y_{r}\right)$ a system of elements of $S$ such that for all $j, 1 \leq j \leq r$ either $\left(\left(y_{1}, \ldots, y_{j-1}\right): y_{j}\right)=\left(y_{1}, \ldots, y_{j}\right)$ and $y_{j}^{2}=0$ holds, or $y_{j}$ is regular on $S /\left(y_{1}, \ldots, y_{j-1}\right)$. Let $S_{u|j|}:=S /\left(y_{1}^{u}, \ldots, y_{j}^{u}\right), u \in$ $\mathbb{N}, 1 \leq j \leq r, S_{1}:=S_{1|r|}=S /(y), e$ an integer, $1 \leq e \leq r, A \in \mathcal{C}_{K}$ and $L$ an $\left(S_{2|e|}\right)_{A}$-module from Lift $\left(\left(S_{2|e|}\right)_{A}, S_{1}\right)$. Then there exists a module $Q \in \operatorname{Lift}\left(S_{A}, S_{1}\right)$ such that $M:=\left(S_{1|e|}\right)_{A} \otimes\left(S_{2|e|}\right)_{A} L$ is a direct summand in $\left(S_{1|e|}\right)_{A} \otimes_{S_{A}} Q$.

Proof: By 1.11 ii), iii) we have $L \in \operatorname{Lift}\left(S_{2|e|}, S_{1|e|}\right)$ and $\bar{M}:=K \otimes_{A} M \in \operatorname{Lift}\left(S_{1|e|}, S_{1}\right)$. Then we have $L \in \operatorname{Lift}\left(\left(S_{2|e|}\right)_{A},\left(S_{1|e|}\right)_{A}\right)$ and $M$ is flat over $A$ by Lemma 1.12. Now we follow the proof of [Po2] (1.16) in the case $S_{A}, y, L, e, r$. Clearly, for all $j, 1 \leq j \leq r$ we have either $\left(\left(y_{1}, \ldots, y_{j-1}\right) S_{A}: y_{j}\right)_{S_{A}}=\left(y_{1}, \ldots, y_{j}\right) S_{A}$ and $y_{j}^{2}=0$ in $A$, or $y_{j}$ is regular on $S_{A} /\left(y_{1}, \ldots, y_{j-1}\right)$ by flatness. Let $e=1$. If $\left(0: y_{1}\right)=\left(y_{1}\right), y_{1}^{2}=0$ then there is nothing to show. If $y_{1}$ is regular on $S_{A}$, then as in [ADS] (3.2) we may take $Q$ to be the first syzygy $\Omega_{S_{A}}^{1}(M) \in \operatorname{Lift}\left(S_{A},\left(S_{1|1|}\right)_{A}\right)$ of $M$ over $S_{A}$ which is clearly flat over $A$. Thus $Q \in \operatorname{Lift}\left(S_{A}, S_{1|1|}\right)$ by 1.10 and $\left.1.11 \mathrm{i}\right)$. The first syzygy $\Omega_{S}^{1}(\bar{M})$ of $\bar{M}$ over $S$ is isomorphic with $K \otimes_{A} Q$ since $M$ is flat over $A$. It follows $K \otimes_{A} Q \in \operatorname{Lift}\left(S_{1|1|}, S_{1}\right)$ since $\bar{M} \in \operatorname{Lift}\left(S_{1|1|}, S_{1}\right)$ and so $Q \in \operatorname{Lift}\left(S_{A}, S_{1}\right)$ by $\left.1.11 \mathrm{i}\right)$.

Suppose that $e>1$. Using $e=1$ in the case

$$
\left(S_{2|e-1|}\right)_{A} \rightarrow\left(S_{2|e|}\right)_{A} \rightarrow\left(\left(S_{2|e|}\right)_{A} / y_{e}\left(S_{2|e|}\right)_{A}\right) \rightarrow S_{1}
$$

there exists a module $Q^{\prime} \in \operatorname{Lift}\left(\left(S_{2|e-1|}\right)_{A}, S_{1}\right)$ such that $L / y_{e} L$ is a direct summand in $Q^{\prime} / y_{e} Q^{\prime}$. Applying induction hypothesis (case $\left.e-1\right)$ to $Q^{\prime}$ there exists $Q \in \operatorname{Lift}\left(S_{A}, S_{1}\right)$ such that $\left(S_{1|e-1|}\right)_{A} \otimes_{\left(S_{2|e-1|}\right)_{A}} Q^{\prime} \cong Q^{\prime} /\left(y_{1}, \ldots, y_{e-1}\right) Q^{\prime}$ is a direct summand in the module $\left(S_{1|e-1|}\right)_{A} \otimes_{S_{A}} Q \cong Q /\left(y_{1}, \ldots, y_{e-1}\right) Q$. Thus $M$ is a direct summand in $\left(S_{1|e|}\right)_{A} \otimes_{\left(S_{2|e|}\right)_{A}} Q^{\prime}$ which is also a direct summand in $\left(S_{1|e|}\right)_{A} \otimes_{S_{A}} Q$.

Corollary 1.14 With the notations and assumptions of Proposition 1.8, let $T$ be a finitely generated $\left(R_{1}\right)_{A}$-module which is flat over $A$. If $T \in \operatorname{lift}\left(\left(R_{2}\right)_{A},\left(R_{1}\right)_{A}\right)$ then $T$ is a direct summand of a module of the form $\left(R_{1}\right)_{A} \otimes_{R_{A}} E$ for a certain $E \in \mathcal{D}_{A}$.

Proposition 1.15 With the notations and assumptions of Theorem 1.4 the functor $\left(R_{1}\right)_{A} \otimes_{R_{A}}$ - induces a bijection between the set of isomorphism classes of modules of $\mathcal{D}_{A}$ and the set of isomorphism classes of $\left(R_{1}\right)_{A}$-modules from lift $\left(\left(R_{2}\right)_{A},\left(R_{1}\right)_{A}\right)$ which are flat over $A$.

Proof: The injectivity follows from Proposition 1.8 as in [Po2] (1.3) (see also [DS]) with $R_{A},\left(R_{1}\right)_{A}$, resp. $\mathcal{D}_{A}$ instead of $R, R_{1}$, resp. $\operatorname{MCM}(R)$ (a module splits uniquely into a direct sum of indecomposable $\left(R_{1}\right)_{A}$-modules since $\left(R_{1}\right)_{A}$ is Henselian!).

Now let $T \in \operatorname{lift}\left(\left(R_{2}\right)_{A},\left(R_{1}\right)_{A}\right)$ be a module which is flat over $A$. By Corollary 1.14 there exists a module $E \in \mathcal{D}_{A}$ such that $\left(R_{1}\right)_{A} \otimes_{R_{A}} E \cong T \oplus T^{\prime}$ for a certain $\left(R_{1}\right)_{A}$-module $T^{\prime}$. Express $E, T, T^{\prime}$ as direct sums of indecomposable modules of $\mathcal{D}_{A}, \operatorname{resp} . \operatorname{Mod}\left(R_{1}\right)_{A}$. Since $\left(R_{1}\right)_{A} \otimes_{R_{A}}$ - maps indecomposable modules of $\mathcal{D}_{A}$ in indecomposable $\left(R_{1}\right)_{A}$-modules, we conclude as in [Po2] (1.4) that there exists a direct summand $F$ of $E$ such that $\left(R_{1}\right)_{A} \otimes_{R_{A}} F \cong$ T. Clearly, $F \in \mathcal{D}_{A}$. 
1.16 Proof of Theorem 1.4. Applying Proposition 1.8 and Proposition 1.15 it remains to show that the modules $T$ from $\operatorname{lift}\left(\left(R_{2}\right)_{A},\left(R_{1}\right)_{A}\right)$ which are flat over $A$ are those $\left(R_{1}\right)_{A}$-modules which deform $R_{1}$-modules of type $x_{1} \ldots x_{r} P$, where $P$ is a finitely generated $R_{2}$-module satisfying $(\mathcal{L})$. Since $T$ is flat over $A$ we have $T \in \operatorname{Lift}\left(\left(R_{1}\right)_{A}, R_{1}\right)$ and so $T \cong\left(R_{1}\right)_{A} \otimes_{\left(R_{2}\right)_{A}} L$ for a certain $L \in \operatorname{Lift}\left(\left(R_{2}\right)_{A}, R_{1}\right)$ (see $\left.1.11 \mathrm{i}\right)$ ). By Lemma 1.12 $L \in \operatorname{Lift}\left(\left(R_{2}\right)_{A}, R_{1}\right)$ if and only if $L$ is flat over $A$ and $P:=K \otimes_{A} L$ satisfies $(\mathcal{L})$. But $x_{1} \ldots x_{r} P \cong R_{1} \otimes_{R_{2}} P$ by [Po2] (1.17). Since $L$ is a deformation of $P$ we see that $T$ is a deformation of $x_{1} \ldots x_{r} P$.

Remark 1.17 Let $M \in \operatorname{MCM}(R)$ and $M_{1}:=M / x M$. In spite of Theorem 1.4 the map $\operatorname{Def}_{M}(A) \rightarrow \operatorname{Def}_{M_{1}}(A)$ induced by base change is not a bijection as the following example shows. The reason is that the isomorphisms considered in Theorem 1.4 are isomorphisms as modules but not as deformations (see 1.2).

Example 1.18 Let $R:=K[[z, y]], z^{2}+y^{3}=0, A:=K[\varepsilon], \varepsilon^{2}=0$ and $M:=(z, y) R$. Then $z^{i}$ generates a reduction ideal for all $i \geq 2$ (see for example [Yo] $(6.16),(6.18)$ ). Take $x=z^{4}$ and let $R_{1}:=R /(x), M_{1}:=M / x M$. Then the base change induces an injective map $\eta: \operatorname{Def}_{M}(K[\varepsilon]) \rightarrow \operatorname{Def}_{M_{1}}(K[\varepsilon])$ which is not surjective. Indeed, let $\eta^{\prime}:=\operatorname{Ext}_{R}^{1}(M, M) \rightarrow$ Ext ${ }_{R_{1}}^{1}\left(M_{1}, M_{1}\right)$ be the map induced by base change. Modulo the canonical isomorphisms $\operatorname{Def}_{M}(K[\varepsilon]) \cong \operatorname{Ext}_{R}^{1}(M, M)$, $\operatorname{Def}_{M_{1}}(K[\varepsilon]) \cong \operatorname{Ext}_{R_{1}}^{1}\left(M_{1}, M_{1}\right)$ the maps $\eta, \eta^{\prime}$ coincide. But $\eta^{\prime}$ is injective by [Yo] (6.17) and not surjective since the following extension

$$
0 \rightarrow M_{1} \stackrel{\alpha}{\rightarrow} R_{1} \otimes_{R} N \stackrel{\beta}{\rightarrow} M_{1} \rightarrow 0
$$

is not in $\operatorname{Im} \eta^{\prime}$, where $N:=M \oplus R^{2} /\left\langle\left(z^{3} y, z, y\right),\left(z^{4}, y^{2},-z\right)\right\rangle$, $\alpha$ is given by the inclusion $M \rightarrow M \oplus R^{2}$ and $\beta$ is given by $(w, u, v) \rightarrow u y-v z, w \in M u, v \in R$. Indeed, $R_{1} \otimes_{R} N$ does not have the form $R_{1} \otimes_{R} P, P \in \operatorname{MCM}(R)$ as was shown in [Po2] (1.20), (1.21). 


\section{Matrix factorizations for liftings}

2.1 Let $(\Lambda, m)$ be a Noetherian local ring, $f \in m$ a non-zero divisor of $\Lambda$ and $R=\Lambda /(f)$. A pair of square $d$-matrices $(\phi, \varphi)$ with entries in $\Lambda$ satisfying $\phi \varphi=f I_{d}$, where $I_{d}$ is the $d \times d$ unit matrix, is called a matrix factorization of $f$. A morphism between matrix factorizations $\left(\phi_{1}, \varphi_{1}\right)$ and $\left(\phi_{2}, \varphi_{2}\right)$ is a pair of square $d$-matrices $(\alpha, \beta)$ with $\alpha \phi_{1}=\phi_{2} \beta$. If $\alpha, \beta$ are invertible matrices then we say that $\left(\phi_{1}, \varphi_{1}\right)$ and $\left(\phi_{2}, \varphi_{2}\right)$ are equivalent. Since $f$ is a nonzero divisor we have $\phi \varphi=f I_{d}$ if and only if $\varphi \phi=f I_{d}$. Also we have $\alpha \phi_{1}=\phi_{2} \beta$ if and only if $\beta \varphi_{1}=\varphi_{2} \alpha$ (see [Ei] or [Yo] Ch. 7). We always identify the square matrix $\phi$ with the linear map $\Lambda^{d} \rightarrow \Lambda^{d}$ associated to $\phi$ in the canonical bases.

Let $\mathrm{MF}_{\Lambda}(f)$ be the category of matrix factorizations of $f$ and morphisms between them. Defining

$$
\left(\phi_{1}, \varphi_{1}\right) \oplus\left(\phi_{2}, \varphi_{2}\right)=\left(\left(\begin{array}{ll}
\phi_{1} & 0 \\
0 & \phi_{2}
\end{array}\right),\left(\begin{array}{ll}
\varphi_{1} & 0 \\
0 & \varphi_{2}
\end{array}\right)\right),
$$

we see that $\mathrm{MF}_{\Lambda}(f)$ is an additive category. A matrix factorization $(\phi, \varphi)$ of $f$ is reduced if the entries of $\phi, \varphi$ are non-units.

Let $\operatorname{RMF}_{\Lambda}(f)$ be the category of reduced matrix factorizations and according to [Yo] (7.3) we define

$$
\begin{gathered}
\underline{\operatorname{MF}}_{\Lambda}(f)=\operatorname{MF}_{\Lambda}(f) /\{(1, f)\}, \\
\underline{\operatorname{RF}}_{\Lambda}(f)=\operatorname{MF}_{\Lambda}(f) /\{(f, 1),(1, f)\}
\end{gathered}
$$

(for notations cf. [Yo]). As in [Ei] and [Yo] (7.2) it holds

Lemma 2.2 Let $(\phi, \varphi)$ be a matrix factorization of $f$. Then the sequence

$$
\ldots \rightarrow R^{d} \stackrel{\phi}{\rightarrow} R^{d} \stackrel{\varphi}{\rightarrow} R^{d} \stackrel{\phi}{\rightarrow} R^{d} \rightarrow \ldots
$$

induced by $\phi, \varphi$ is exact.

Proposition 2.3 Let $a \subset m$ be an ideal such that $f \notin a, \bar{R}:=R / a R$ and suppose that $\bar{\Lambda}:=\Lambda / a$ is a discrete valuation ring (in short a DVR). Then

i) if $(\phi, \varphi)$ is a matrix factorization of $f$ then $\operatorname{Tor}_{i}^{R}(\bar{R}, \operatorname{Coker} \phi)=0$ for all $i \geq 1$, that is, Coker $\phi \in \operatorname{Lift}(R, \bar{R})$ (see 1.10),

ii) if $M \in \operatorname{Lift}(R, \bar{R})$ then there exists a matrix factorization $(\phi, \varphi)$ of $f$ such that $M \cong$ Coker $\phi$.

\section{Proof:}

i) Let $\bar{f}, \bar{\phi}, \bar{\varphi}$ be the maps induced by $f, \phi, \varphi$ modulo $a$. Then $(\bar{\phi}, \bar{\varphi})$ is a matrix factorization of $\bar{f}$ and the sequence

$$
\cdots \rightarrow \bar{R}^{d} \stackrel{\bar{\phi}}{\rightarrow} \bar{R}^{d} \stackrel{\bar{\varphi}}{\rightarrow} \bar{R}^{d} \stackrel{\bar{\phi}}{\rightarrow} \bar{R}^{d} \rightarrow \bar{M}:=\operatorname{Coker} \bar{\phi} \rightarrow 0
$$

induced by $(\bar{\phi}, \bar{\varphi})$ is exact (see 2.2). Thus (7) gives a free resolution of $\bar{M}$ over $\bar{R}$. By Lemma 2.2 a free resolution of $M:=\operatorname{Coker} \phi$ is given by

$$
\ldots \rightarrow R^{d} \stackrel{\phi}{\rightarrow} R^{d} \stackrel{\varphi}{\rightarrow} R^{d} \stackrel{\phi}{\rightarrow} R^{d} \rightarrow M \rightarrow 0 .
$$


Since (7) is obtained by tensorizing (8) by $\bar{R}$ over $R$, we see that $\operatorname{Tor}_{i}^{R}(\bar{R}, M)=0$ for all $i \geq 1$.

ii) Let $M \in \operatorname{Lift}(R, \bar{R})$ and $\bar{M}:=\bar{R} \otimes_{R} M$. Then $\operatorname{pd}_{\bar{\Lambda}} \bar{M} \leq 1$ as $\bar{\Lambda}$ is a DVR. But $\bar{M}$ is not free over $\bar{\Lambda}$ because $\bar{f} \bar{M}=0$. Thus, $\operatorname{pd}_{\bar{\Lambda}} \bar{M}=1$.

We have $\operatorname{Tor}_{i}^{\Lambda}(\bar{\Lambda}, M) \cong \operatorname{Tor}_{i}^{R}(\bar{R}, M)=0$ for all $i \geq 1$, the isomorphism follows because tensorizing by $R$ over $\Lambda$ a free resolution of $\bar{\Lambda}$ over $\Lambda$ we still obtain a free resolution of $\bar{R}$ over $R$ ( $f$ is a $\Lambda$ and $\bar{\Lambda}$-regular element!). Thus tensorizing by $\bar{\Lambda}$ a minimal free resolution of $M$ over $\Lambda$ we get a minimal free resolution of $\bar{M}$ over $\bar{\Lambda}$. Hence, $\operatorname{pd}_{\Lambda} M=1$. Let

$$
0 \rightarrow \Lambda^{s} \stackrel{\phi}{\rightarrow} \Lambda^{d} \rightarrow M \rightarrow 0
$$

be a minimal free resolution of $M$ over $\Lambda$ which induces a minimal free resolution of $\bar{M}$ over $\bar{\Lambda}$. Since $\operatorname{rank}_{\bar{\Lambda}} \bar{M}=0$ we obtain $s=d$. As in [Ei] or [Yo] (7.1.2) we see that there exists an endomorphism $\varphi \in \operatorname{End}_{\Lambda}\left(\Lambda^{d}\right)$ such that $\phi \varphi=f 1_{\Lambda^{d}}$. Clearly, $(\phi, \varphi)$ defines a matrix factorization of $f$ such that Coker $\phi=M$.

Corollary 2.4 With the hypothesis and notation of Proposition 2.3 every module from $\operatorname{Lift}(R, \bar{R})$ has a periodic free resolution with periodicity 2.

2.5 Matrix Factorization Theorem. Let $a \subset m$ be an ideal such that $f \notin a, \bar{R}:=$ $R / a R$ and suppose that $\Lambda / a$ is a DVR. Then the functor Coker : $\operatorname{MF}_{\Lambda}(f) \rightarrow \operatorname{Lift}(R, \bar{R})$ given by $(\phi, \varphi) \rightarrow$ Coker $\phi$ induces an equivalence of categories:

$$
\underline{\operatorname{MF}}_{\Lambda}(f) \stackrel{\sim}{\rightarrow} \operatorname{Lift}(R, \bar{R}) .
$$

Moreover, this induces an equivalence:

$$
\underline{\operatorname{RMF}}_{\Lambda}(f) \stackrel{\sim}{\rightarrow} \underline{\operatorname{Lift}}(R, \bar{R})
$$

where $\underline{\operatorname{Lift}}(R, \bar{R})=\operatorname{Lift}(R, \bar{R}) /\{R\}$, (notations cf. [Yo]).

The proof can be given exactly as in [Ei] or [Yo] (7.4) using Proposition 2.3.

Corollary 2.6 With the hypothesis of Theorem 2.5 the functor Coker yields a bijection preserving the indecomposability between the set of equivalence classes of reduced matrix factorizations of $f$ and the set of isomorphism classes of modules from Lift $(R, \bar{R})$ which have no free summands.

Corollary 2.7 With the hypothesis of Theorem 2.5, let $(\phi, \varphi)$ be a reduced matrix factorization of $f$ and $M=\operatorname{Coker}(\phi, \varphi)$. If $M$ is indecomposable then the first syzygy $\Omega_{\Lambda}^{1}(M)$ of $M$ over $\Lambda$ is also indecomposable and $\Omega_{\Lambda}^{1}(M) \cong \operatorname{Coker}(\varphi, \phi)$.

The proofs of these Corollaries follow [Yo] (7.6), (7.7). 
Theorem 2.8 Let $(B, \wp, K)$ be a Noetherian local ring, $\Lambda=B[[X]], X=\left(X_{1}, \ldots, X_{r}\right)$ some indeterminates, $f \in \Lambda$ be a non-unit formal power series which is not in $\wp \Lambda, \mathcal{R}:=$ $\Lambda /(f), R:=K \otimes_{B} \mathcal{R}$ and $\mathcal{D}_{B}^{R}$ the category of all finitely generated $\mathcal{R}$-modules $E$ which are flat over $B$ and such that $K \otimes_{B} E \in M C M(R)$. Suppose that $\mathcal{R}$ is flat over $B$. Then Coker induces an equivalence of the categories:

$$
\underline{M F}_{\Lambda}(f) \stackrel{\sim}{\rightarrow} \mathcal{D}_{B}^{R} .
$$

Moreover, this induces an equivalence:

$$
\underline{R M F}_{\Lambda}(f) \stackrel{\sim}{\rightarrow} \mathcal{D}_{B}^{R} /\{\mathcal{R}\}
$$

Proof: If $f \notin a:=\left(\wp, X_{1}, \ldots, X_{r-1}\right) \Lambda$ then apply Theorem 2.5 for $\Lambda, \mathcal{R}, a$. Clearly $\operatorname{Lift}(\mathcal{R}, R)$ is exactly the category of all finitely generated $\mathcal{R}$-modules flat over $B$. Indeed, we have $\operatorname{Tor}_{i}^{\mathcal{R}}(R, E) \cong \operatorname{Tor}_{i}^{B}(K, E)$ for all $\mathcal{R}$-modules $E$, because $\mathcal{R}$ is flat over $B$ and $R=K \otimes_{B} \mathcal{R}$. Thus, $E \in \operatorname{Lift}(\mathcal{R}, R)$ if and only if $\operatorname{Tor}_{i}^{B}(K, E)=0$ for all $i \geq 1$, that is if and only if $E$ is flat over $B$ by the local flatness critierium (see [Ma] (20.C)). Since $X_{1}, \ldots, X_{r-1}$ defines a system of parameters in $R$, thus a regular sequence in $R(R$ is a hypersurface over $K$ and so a Cohen-Macaulay $\operatorname{ring})$, we have $E \in \operatorname{Lift}(\mathcal{R}, \mathcal{R} / a \mathcal{R})$ if and only if $E \in \operatorname{Lift}(\mathcal{R}, R)$ and $X_{1}, \ldots, X_{r-1}$ is regular on $\bar{E}:=K \otimes_{B} E=R \otimes_{\mathcal{R}} E$ (1.11 ii)). Thus, $\operatorname{Lift}(\mathcal{R}, \mathcal{R} / a \mathcal{R})=\mathcal{D}_{B}^{R}$. If $f \in a$ then modulo an automorphism of type $X_{i} \rightarrow X_{i}+X_{r}^{e_{i}}, i<r, X_{r} \rightarrow X_{r}$ we may suppose $f \notin a$.

Corollary 2.9 With the hypothesis of Theorem 2.8 the functor Coker yields a bijection preserving the indecomposability between the set of equivalence classes of reduced matrix factorizations of $f$ over $\Lambda$ and the set of isomorphism classes of modules from $\mathcal{D}_{B}^{R}$ which have no free summands.

Remark 2.10 If $B=K$ then Theorem 2.8 is exactly the Eisenbud Matrix Factorization Theorem (see [Ei], Section 6).

Theorem 2.11 With the hypothesis and notation of Theorem 2.8, let $S:=$ $\Lambda /\left(X_{1}^{2}, \ldots, X_{r-1}^{2}\right), \mathcal{H}:=S /(f), H:=K \otimes_{B} \mathcal{H}$ and $\mathcal{L}_{B}^{H}$ be the category of all finitely generated $\mathcal{H}$-modules $E$ which are flat over $B$ and such that $\bar{E}:=K \otimes_{B} E$ satisfies

$$
\left(\left(X_{1}, \ldots, X_{j-1}\right) \bar{E}: X_{j}\right)_{E}=\left(X_{1}, \ldots, X_{j}\right) \bar{E}
$$

for every $j, 1 \leq j<r$. Suppose that $\mathcal{H}$ is flat over $B$ and $f \notin\left(X_{1}, \ldots, X_{r-1}\right)$. Then Coker induces an equivalence of the categories

$$
\underline{M F}_{S}(f) \stackrel{\sim}{\rightarrow} \mathcal{L}_{B}^{H} .
$$

Moreover, this induces an equivalence:

$$
\underline{R M F}_{S}(f) \stackrel{\sim}{\rightarrow} \mathcal{L}_{B}^{H} /\{\mathcal{H}\}
$$


Proof: Clearly $\left\{X_{1}, \ldots, X_{r-1}, f\right\}$ forms a system of parameters in $\Lambda$ and thus a regular system of elements in $\Lambda$. Then $\left\{X_{1}, \ldots, X_{r-1}\right\}$ forms a regular system of elements in $\Lambda /(f)$ and so $H$ satisfies $(\mathcal{L})$ above. Since $\mathcal{H}$ is flat over $B$ we have $\mathcal{H} \in \mathcal{L}_{B}^{H}$. Hence, $\mathcal{L}_{B}^{H}=$ Lift $(\mathcal{H}, \mathcal{H} / a \mathcal{H})$, where $a=\left(\wp, X_{1}, \ldots, X_{r-1}\right) S$ (see 1.11 iii) and the proof of 2.8). Now it is enough to apply Theorem 2.5 for $S, a, f, \mathcal{H}$.

Corollary 2.12 With the hypothesis and notation of Theorem 2.11, the functor Coker yields a bijection preserving the indecomposability between the set of equivalence classes of reduced matrix factorizations of $f$ over $S$ and the set of isomorphism classes of modules from $\mathcal{L}_{B}^{H}$ which have no free summands.

2.13 Keeping the hypothesis and notation of Theorem 2.8 , let $\bar{\Lambda}:=K \otimes_{B} \Lambda$ and $\bar{f} \in \bar{\Lambda}$ be the element induced by $f$. Let $(\rho, \eta)$ be a matrix factorization of $\bar{f}$ over $\bar{\Lambda}$. A deformation of $(\rho, \eta)$ over $B$ is a matrix factorization $(\phi, \psi) \in \underline{M F}_{\Lambda}(f)$ of $f$ over $\Lambda$ together with an isomorphism $(\alpha, \beta):(\rho, \eta) \rightarrow(K \otimes \phi, K \otimes \psi)$. Two deformations $\left(\phi_{1}, \psi_{1}\right),\left(\phi_{2}, \psi_{2}\right)$ of $(\rho, \eta)$ over $B$ are isomorphic if an isomorphism $(\lambda, \tau):\left(\phi_{1}, \psi_{1}\right) \rightarrow\left(\phi_{2}, \psi_{2}\right)$ exists such that the following diagram commutes in $\mathrm{MF}_{\Lambda}(f)$

$$
\begin{array}{ccc}
(\rho, \eta) & \stackrel{\sim}{\rightarrow} & \left(K \otimes \phi_{1}, K \otimes \psi_{1}\right) \\
\| & & \downarrow \\
(\rho, \eta) & \stackrel{\sim}{\rightarrow} & \left(K \otimes \phi_{2}, K \otimes \psi_{2}\right)
\end{array}
$$

where the second vertical map is induced by $(\lambda, \tau)$. $\operatorname{Def}_{(\rho, \eta)}^{\Lambda}(B)$ denotes the set of isomorphism classes of deformations of $(\rho, \eta)$ over $B$.

Now let $M$ be a finitely generated $R$-module. A finitely generated $\mathcal{R}$-module $E$ which is flat over $B$ together with an isomorphism $\mathcal{X}: M \stackrel{\sim}{\rightarrow} K \otimes_{B} E$ forms a deformation of $M$ over $B$. Two deformations $E_{1}, E_{2}$ of $M$ over $B$ are isomorphic if an isomorphism $\theta: E_{1} \rightarrow E_{2}$ exists such that the following diagram commutes

$$
\begin{array}{ccc}
M & \stackrel{\sim}{\rightarrow} & K \otimes E_{1} \\
\| & & \downarrow \\
M & \stackrel{\sim}{\rightarrow} & K \otimes E_{2}
\end{array}
$$

where the second vertical map is given by $\theta$. The set of isomorphism classes of deformations of $M$ over $B$ is denoted by $\operatorname{Def}_{M}^{\mathcal{R}}(B)$.

Theorem 2.14 Keeping the hypothesis and notation of Theorem 2.8, let $(\rho, \eta)$ be a matrix factorization of $\bar{f}$ over $\bar{\Lambda}$ and $M:=$ Coker $\rho$. Then the functor Coker yields a bijection $\omega: \operatorname{Def}_{(\rho, \eta)}^{\Lambda}(B) \rightarrow \operatorname{Def}_{M}^{\mathcal{R}}(B)$.

The proof follows since Coker induces the equivalences $\underline{\operatorname{MF}}_{\Lambda}(f) \stackrel{\sim}{\rightarrow} \mathcal{D}_{B}^{R}, \underline{\operatorname{MF}}_{\bar{\Lambda}}(f) \stackrel{\sim}{\rightarrow}$ $\operatorname{MCM}(R)$. Note that the injectivity of $\omega$ holds only because we work modulo $\{(1, f)\}$ (see the proof of [Yo] (7.4)).

Example 2.15 Let $R:=K[[Z, Y]] /\left(Z^{2}+Y^{3}\right), A:=K[[t]], R_{A}:=R[[t]]$ and $M:=$ $\left(Z Y, Y^{2}\right) R$ be the (only) non-trivial indecomposable maximal Cohen-Macaulay $R$-module. 
$N:=\left(Z Y, Y^{2}+t Z\right) R_{A} \subset R_{A}$ is a deformation of $M$ over $A$. The corresponding matrix factorization is

$$
\left(\left(\begin{array}{cc}
Z-t Y & Y+t^{2} \\
Y^{2} & -Z-t Y
\end{array}\right), \quad\left(\begin{array}{cc}
Z+t Y & Y+t^{2} \\
Y^{2} & -Z+t Y
\end{array}\right)\right) .
$$

Now consider a deformation of $R$ over $A$ defined by $\mathcal{R}:=K[[Z, Y, t]] /\left(Z^{2}+Y^{3}+t Y^{2}\right)$ and the module $M:=\left(Y^{2},-Z\right) R_{A}$. The corresponding matrix factorization of $M$ over $\mathcal{R}$ is

$$
\left(\left(\begin{array}{cc}
Z & Y+t \\
Y^{2} & -Z
\end{array}\right), \quad\left(\begin{array}{cc}
Z & Y+t \\
Y^{2} & -Z
\end{array}\right)\right) .
$$

Example 2.16 Let $R:=K[[X, Y]] /\left(X^{3}+Y^{4}\right)$. We are interested in studying the deformations of the normalization of $R$. To have a nice description (all modules are embedded in $R$ with fixed colength) one has to identify the normalization with the ideal $\left(X Y^{2}, Y^{3}, X^{2} Y\right) \subset R$. The other rank one modules are then $\left(X Y, X^{3}\right),\left(X^{2}, X Y^{2}\right),\left(X^{2}, Y^{3}\right)$ and $\left(Y^{2}\right)$ (see $[\mathrm{PS}]$ or $\left.[\mathrm{GK}]\right)$. The normalization can be deformed into $\left(X Y, X^{3}\right)$ resp. $\left(X^{2}, X Y^{2}\right)$ using the family $\left(X Y^{2}, Y^{3}+u X^{2}+v X Y, X^{2} Y\right)$ with the parameters $u, v$. The corresponding matrix factorization is given by

$$
\begin{gathered}
\left(\begin{array}{ccc}
Y+u v & -v Y & X \\
-X-u Y & Y^{2} & 0 \\
v+u^{2} & X-u Y & -Y
\end{array}\right), \\
\left(\begin{array}{ccc}
Y^{3} & -X^{2}+u X Y+v Y^{2} & X Y^{2} \\
X Y+u Y^{2} & Y^{2}+u^{2} X+v X+u v Y & X^{2}+u X Y \\
X^{2}+v Y^{2} & X Y-u Y^{2}+u v X+v^{2} Y & -Y^{3}+v X Y
\end{array}\right) .
\end{gathered}
$$

Similarly, one can obtain the matrix factorization for the deformation $\left(X Y^{2}+v X^{2}, Y^{3}+\right.$ $\left.u X^{2}, X^{2} Y\right)$ and the smoothing family. 


\section{Knörrer's Periodicity Theorem for liftings}

3.1 Let $(\Lambda, m, K)$ be a Noetherian, Henselian, local ring, $f \in m$ a non-zero divisor of $\Lambda$, $R=\Lambda /(f), a \subset m$ an ideal of $\Lambda$ such that $f \notin a, \bar{R}:=R / a R$ and $\Lambda^{*}:=\Lambda[[U]]$. Suppose that $\Lambda / a$ is a DVR. Let $\lambda, \mu$ be two elements from $\Lambda, \lambda \in m$ and $R^{*}:=\Lambda^{*} /\left(f-\lambda U+\mu U^{2}\right)$. We have $\bar{R} \cong R^{*} /(a, U) R^{*}$.

Lemma 3.2 The following statements are equivalent for a finitely generated $R^{*}$-module $N$ :

i) $N \in \operatorname{Lift}\left(R^{*}, \bar{R}\right)$,

ii) $N \in \operatorname{Lift}\left(R^{*}, R^{*} / a R^{*}\right)$ and $U$ is regular on $N / a N$,

iii) $U$ is regular on $N$ and $N / U N \in \operatorname{Lift}(R, \bar{R})$.

Proof: First note that $U$ is a non-zero divisor on $R^{*}$ and $R^{*} / a R^{*} \cong \Lambda / a[[U]] /\left(f-\lambda U+\mu U^{2}\right)$. Indeed, if $U g=\left(f-\lambda U+\mu U^{2}\right) h$ for some non-zero formal power series $g, h \in \Lambda / a[[U]]$, then we may choose $h$ with $h(U=0) \neq 0$. Thus, $f h(U=0)=0$, which is not possible since $f$ is a non-zero divisor in $\Lambda / a$. Hence, $U$ is regular on $R^{*} / a R^{*}$.

ii) $\Rightarrow$ i) and iii) $\Rightarrow$ i) follow easily from 1.11 i), ii) and i) $\Rightarrow$ ii) follows from 1.11 ii) since $U$ is regular on $R^{*} / a R^{*}$. If i) holds then $N$ has a periodic free resolution over $R^{*}$ with periodicity 2 (see 2.4). In particular, $N$ is a submodule in a free $R^{*}$-module. As $U$ is regular on $R^{*}$ it follows that $U$ is also regular on $N$. Since $N \in \operatorname{Lift}\left(R^{*}, \bar{R}\right)$ (ii) implies i)!) it follows that $N / U N \in \operatorname{Lift}(R, \bar{R})$ by $1.11 \mathrm{i})$.

3.3 Let $N \in \operatorname{Lift}\left(R^{*}, \bar{R}\right)$. By Lemma $3.2 U$ is a non-zero divisor on $N / a N$. Since the ring $R^{*} / a R^{*}=\Lambda / a[[U]] /\left(f-\lambda U+\mu U^{2}\right)$ is Cohen-Macaulay of dimension one, we note that $N / a N$ is a maximal Cohen-Macaulay module over $R^{*} / a R^{*}$. If $\mu$ is a unit then $R^{*} / a R^{*}$ is finite over $\Lambda / a$ and $N / a N$ must be free over $\Lambda / a$. We have $\operatorname{Tor}_{i}^{\Lambda}(\Lambda / a, N) \cong \operatorname{Tor}_{i}^{R^{*}}\left(R^{*} / a R^{*}, N\right)$ for all $i \geq 1$ since $R^{*}$ is free over $\Lambda$. But by $3.2 N \in \operatorname{Lift}\left(R^{*}, R^{*} / A R^{*}\right)$ and it follows that $\operatorname{Tor}_{i}^{\Lambda}(\Lambda / a, N)=0$ for all $i \geq 1$, that is $N \in \operatorname{Lift}(\Lambda, \Lambda / a)$. As the liftings of free modules are free, we conclude that $N$ must be free over $\Lambda$.

Let $e_{1}, \ldots, e_{t}$ be a basis in $N$ over $\Lambda$. Then the multiplication with $U$ on $N$ is given by a square $t$-matrix $\phi$ with entries in $\Lambda$ and with the property $-\lambda \phi+\mu \phi^{2}=-f I_{t}$.

Lemma 3.4 Suppose $\mu=1$. Let $N \in \operatorname{Lift}\left(R^{*}, \bar{R}\right)$ and $\phi$ be a square $t$-matrix over $\Lambda$ given by the action of $U$ on $N$. Then $\left(U I_{t}-\phi,(U-\lambda) I_{t}+\phi\right)$ lies in $M F_{\Lambda^{*}}\left(f-\lambda U+U^{2}\right)$ with Coker $\left(U I_{t}-\phi,(U-\lambda) I_{t}+\phi\right) \cong N$. Similarly, $(\phi, \lambda-\phi)$ is in $M F_{\Lambda}(f)$ such that $\operatorname{Coker}(\phi, \lambda-\phi) \cong N / U N$.

Proof: We follow [Yo] (12.2). Clearly $(\phi, \lambda-\phi) \in \mathrm{MF}_{\Lambda}(f)$ and $\left(U I_{t}-\phi,(U-\lambda) I_{t}+\phi\right) \in$ $\operatorname{MF}_{\Lambda^{*}}\left(f-\lambda U+U^{2}\right)$. Let $N^{\prime}:=\operatorname{Coker}\left(U I_{t}-\phi\right), p: \Lambda^{* t} \rightarrow N^{\prime}$ be the canonical surjection and $e_{1}, \ldots, e_{t}$ the canonical basis in $\Lambda^{* t}$. We claim that $\bar{e}:=\left\{p\left(e_{1}\right), \ldots, p\left(e_{t}\right)\right\}$ forms a basis in $N^{\prime}$ over $\Lambda$. Indeed, since $U e_{i} \equiv \phi\left(e_{i}\right) \bmod \operatorname{Im}\left(U I_{t}-\phi\right)$ it follows $U p\left(e_{i}\right)=\phi\left(p\left(e_{i}\right)\right)$ that $\bar{e}$ generates $N^{\prime}$ over $\Lambda$. If $\sum_{i=1}^{t} \gamma_{i} p\left(e_{i}\right)=0$ for some $\gamma_{i} \in \Lambda$ then $\sum_{i=1}^{t} \gamma_{i} e_{i} \in \operatorname{Im}\left(U I_{t}-\phi\right)$, that is there exist $\tau_{j} \in \Lambda$ such that $\sum_{i=1}^{t} \gamma_{i} e_{i}=\sum_{i=1}^{t} \tau_{j}\left(U e_{j}-\phi\left(e_{j}\right)\right)$. Identifying the coefficients of $U$ above we obtain $\sum_{j=1}^{t} \tau_{j} e_{j}=0$, and so $\tau_{j}=0$. Thus, $\sum_{i=1}^{t} \gamma_{i} e_{i}=0$ which 
gives $\gamma_{i}=0$. Hence, $\bar{e}$ is a basis of $N^{\prime}$. Since the multiplication of $U$ on $N^{\prime}$ must act like $\phi$ on $\bar{e}$ it follows that $N \cong N^{\prime}$ as $\Lambda^{*}$-modules and so as $R^{*}$-modules as well. Then $N / U N \cong R \otimes_{R^{*}}$ Coker $\left(U I_{t}-\phi\right) \cong \operatorname{Coker}(-\phi)=$ Coker $\phi$ which ends the proof.

Lemma 3.5 Let $M \in \operatorname{Lift}(R, \bar{R})$ be a module without free summands and $(\phi, \varphi)$ a reduced matrix factorization of $f$ with $M=\operatorname{Coker}(\phi, \varphi)$ (see 2.2). Then

$$
\theta=\left(\left(\begin{array}{cc}
\varphi & -U \\
\mu U-\lambda & \phi
\end{array}\right), \quad\left(\begin{array}{cc}
\phi & U \\
\lambda-\mu U & \varphi
\end{array}\right)\right)
$$

is an element in $M F_{\Lambda^{*}}\left(f-\lambda U+\mu U^{2}\right)$ whose cokernel is the reduced first syzygy $\tilde{\Omega}_{R^{*}}^{1}(M)$ (that is the non-free part of the first syzygy) of $M$ over $R^{*}$.

Proof: We follow [Yo] (12.3). Let $\sigma$ be the first matrix from $\theta$. We shall show that the following sequence

$$
R^{* 2 t} \stackrel{\sigma}{\rightarrow} R^{* 2 t} \stackrel{\left(\phi, U I_{t}\right)}{\longrightarrow} R^{* t} \rightarrow M \rightarrow 0
$$

is exact. Clearly, (10) is a complex and $R^{* t} / \operatorname{Im}\left(\phi, U I_{t}\right)=R^{* t} /\left(U R^{* t}+\operatorname{Im} \phi\right) \cong R^{t} / \operatorname{Im} \phi=$ $M$. If $\alpha, \beta \in R^{* t}$ satisfies $\phi(\alpha)=-U \beta$ then $\alpha \bmod U$ belongs to the kernel of the map $R^{t} \stackrel{\phi}{\rightarrow} R^{t}$. As $\phi, \varphi$ defines the exact sequence from Lemma 2.2 there exists $\gamma \in R^{* t}$ such that $\alpha \equiv \varphi(\gamma) \bmod U$, that is $\alpha=\varphi(\gamma)-U \tau$ for a certain $\tau \in R^{* t}$. Then $U \beta=-\phi(\alpha)=$ $-\phi \varphi(\gamma)+U \phi(\tau)=\left(\mu U^{2}-\lambda U\right) \gamma+U \phi(\tau)$ because $\phi \varphi=f I_{t}=\left(\lambda U-\mu U^{2}\right) I_{t}$. Hence, $\beta=(\mu U-\lambda) \gamma+\phi(\tau)$ and

$$
\left(\begin{array}{l}
\alpha \\
\beta
\end{array}\right)=\left(\begin{array}{cc}
\varphi & -U \\
\mu U-\lambda & \phi
\end{array}\right)\left(\begin{array}{l}
\gamma \\
\tau
\end{array}\right)
$$

follows.

Consequently (10) is exact and, therefore,

$$
\text { Coker } \sigma \cong \tilde{\Omega}_{R^{*}}^{1}(M) \oplus L,
$$

where $L$ is a free $R^{*}$-module. Since $\theta$ is a reduced matrix factorization $(\lambda \in m !)$ we see that its cokernel has no free summands (see 2.6) and so $L=0$.

Proposition 3.6 Let $M \in \operatorname{Lift}(R, \bar{R})$ and $N \in \operatorname{Lift}\left(R^{*}, \bar{R}\right)$. Suppose $M$ (resp. $N$ ) has no free $R$-summands (resp. $R^{*}$-summands). Then

i) $R \otimes_{R^{*}} \tilde{\Omega}_{R^{*}}^{1}(M) \cong M \oplus \Omega_{R}^{1}(M)$ if $\lambda=0$, where $\Omega_{R}^{1}(M)$ denotes the first syzygy of $M$ over $R$,

ii) $\Omega_{R^{*}}^{1}(N / U N) \cong N \oplus \Omega_{R^{*}}^{1}(N)$ if $U \operatorname{Ext}_{R^{*}}^{1}\left(N, \Omega_{R^{*}}^{1}(N)\right)=0$. 


\section{Proof:}

i) Let $(\phi, \varphi)$ be a reduced matrix factorization of $f$ over $\Lambda$ such that Coker $\phi=M$. According to Lemma $3.5 \tilde{\Omega}_{R^{*}}^{1}(M)$ is the kernel of the matrix factorization

$$
\theta=\left(\left(\begin{array}{cc}
\varphi & -U \\
\mu U-\lambda & \phi
\end{array}\right),\left(\begin{array}{cc}
\phi & U \\
\lambda-\mu U & \varphi
\end{array}\right)\right)
$$

Thus,

$$
\begin{gathered}
R \otimes_{R^{*}} \tilde{\Omega}_{R^{*}}^{1}(M) \cong \operatorname{Coker} R \otimes_{R^{*}}\left(\begin{array}{cc}
\varphi & -U \\
\mu U-\lambda & \phi
\end{array}\right)= \\
\quad \text { Coker }\left(\begin{array}{rr}
\varphi & 0 \\
-\lambda & \phi
\end{array}\right) \cong M \oplus \Omega_{R}^{1}(M)
\end{gathered}
$$

if $\lambda=0$.

ii) Let $s$ be the minimal number of generators of $N$ over $R^{*}$. We have the following commutative diagram

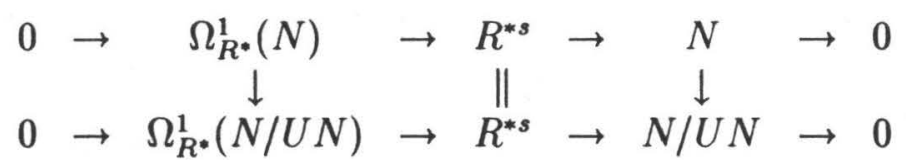

where the rows are exact and the third vertical map is the canonical surjection. The Snake Lemma yields that the cokernel of the first vertical map is $U N$, that is, the sequence

$$
0 \rightarrow \Omega_{R^{*}}^{1}(N) \rightarrow \Omega_{R^{*}}^{1}(N / U N) \rightarrow U N \rightarrow 0
$$

is exact. We have the following commutative diagram

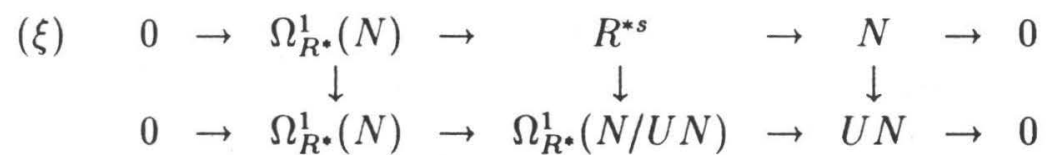

where the rows are exact and the vertical maps are multiplications by $U\left(U R^{* s} \subset\right.$ $\Omega_{R^{*}}^{1}(N / U N) \subset R^{* s}$ !). As $U$ is regular on $N$ according to Lemma 3.2 we see that the third vertical map is bijective. Then (11) gives in $\operatorname{Ext}_{R^{*}}^{1}\left(N, \Omega_{R^{*}}^{1}(N)\right)$ the element $U \xi$, where $\xi$ is the top row from the above diagram. But $U \xi=0$ by assumptions and so (11) splits.

Corollary 3.7 Let $R^{\prime}:=\Lambda[[U, V]] /(f+U V), T:=U-V, M \in \operatorname{Lift}(R, \bar{R})$ and $N \in$ Lift $\left(R^{\prime}, \bar{R}\right), R \cong R^{\prime} /(U, V) R^{\prime}$. Suppose $M$ (resp. $N$ ) has no free $R$-summands (resp. $R^{*}$ summands). Then

i) $R \otimes_{R[[T]]}\left(\tilde{\Omega}_{R[[T]]}^{1}(M)\right) \cong M \oplus \Omega_{R}^{1}(M)$,

ii) $\Omega_{R[[T]]}^{1}(N /(U, T) N) \cong N / U N \oplus \Omega_{R[[T]]}^{1}(N / U N)$, $\Omega_{R^{\prime}}^{1}(N / U N) \cong N \oplus \Omega_{R^{\prime}}^{1}(N)$. 
Proof: i) follows from Proposition 3.6 i) for $\lambda=\mu=0$. For ii) we need the following

Lemma $3.8(U, V) \operatorname{Ext}_{R^{\prime}}^{1}(N,-)=0$ for all $N \in \operatorname{Lift}\left(R^{\prime}, \bar{R}\right)$.

The second isomorphism from ii) follows by Proposition 3.6 ii) applied for $\lambda:=T, \mu:=1$ with the help of the above Lemma. Now, let $P$ be an arbitrary $R[[T]]$-module. By Lemma 3.8 we have $T \operatorname{Ext}_{R^{\prime}}^{1}(N, P)=0$. But $\operatorname{Ext}_{R^{\prime}}^{1}(N, P) \cong \operatorname{Ext}_{R^{\prime} /(U)}^{1}(N / U N, P)$ since $U$ is regular on $N, R^{\prime}$ and $U P=0$. Hence, for all $R[[T]]$-modules $P$ we have $T \operatorname{Ext}_{R^{\prime} /(U)}^{1}(N / U N, P)=$ 0 ; in particular for $P:=\Omega_{R[[T]]}^{1}(N / U N)$. Then the first isomorphism from ii) follows by Proposition 3.6 ii) applied for $\lambda=\mu=0$.

3.9 Proof of Lemma 3.8. Let $\varepsilon \in \Lambda$ be a unit, $T_{\varepsilon}:=\varepsilon U-V$ and $N \in \operatorname{Lift}\left(R^{\prime}, \bar{R}\right)$. Then $R^{\prime} \cong \Lambda\left[\left[T_{\varepsilon}, U\right]\right] /\left(f-T_{\varepsilon} U+\varepsilon U^{2}\right)$ is finite over $C_{\varepsilon}:=\Lambda\left[\left[T_{\varepsilon}\right]\right]$. Since $N$ is free over $C_{\varepsilon}$ (see 3.3 for the case $\lambda:=T_{\varepsilon}, \mu:=\varepsilon$ ),

$$
\mathcal{N}_{R^{\prime} / C_{\boldsymbol{c}}} \operatorname{Ext}_{R^{\prime}}^{1}(N,-)=0
$$

follows, as we have seen in the proof of 1.7. Note that $R^{\prime} \otimes_{C_{e}} R^{\prime} \cong R^{\prime}\left[\left[U^{\prime}\right]\right] /\left(f-T_{\varepsilon} U^{\prime}+\varepsilon U^{\prime 2}\right)$, $U^{\prime}$ being a new indeterminate. The kernel of the map $\rho: R^{\prime} \otimes_{C_{\varepsilon}} R^{\prime} \rightarrow R^{\prime}, y \otimes y^{\prime} \rightarrow y y^{\prime}$ is $\left(U-U^{\prime}\right) R^{\prime} \otimes_{C_{e}} R^{\prime}$. Clearly we have $\left(U-U^{\prime}\right)\left(T_{\epsilon}-\varepsilon U-\varepsilon U^{\prime}\right)=0$ and so $\mathcal{N}_{R^{\prime} / C_{\epsilon}}=$ $\rho\left(\operatorname{Ann}_{R^{\prime} \otimes_{C_{\varepsilon}} R}\left(U-U^{\prime}\right)\right)$ contains $T_{\varepsilon}-2 \varepsilon U=-V-\varepsilon U$. If $K$ has at least three elements then we can find a unit $\varepsilon_{1} \in \Lambda$ such that $\varepsilon_{2}:=\varepsilon_{1}+1$ is still a unit. Then $U=-V-\varepsilon_{1}-\left(-V-\varepsilon_{2} U\right)$ (and $V$ as well) annihilates $\operatorname{Ext}_{R^{\prime}}^{1}(N,-)$ by (12)). If $K$ has only two elements, that is $K=\mathbb{Z}_{2}$ then let $\Gamma:=\Lambda[X]_{m[X]}, \tilde{R}:=\Gamma /(f), \tilde{R}^{\prime}:=\Gamma[[U, V]] /(f+U V)$ and $\tilde{N}:=\tilde{R}^{\prime} \otimes_{R^{\prime}} N$. Clearly the residue field $\mathbb{Z}_{2}(X)$ of $\Gamma$ is infinite. Then $(U, V) \operatorname{Ext}_{\tilde{R}^{\prime}}{ }^{\prime}(\tilde{N},-)=0$ and thus, by faithful flatness, we are finished.

Remark 3.10 The proof of $3.7 \mathrm{ii})$ in the case of [Kn] or [Yo] (12.4) uses directly the Lemmas 3.4, 3.5. Combining these Lemmas we can also see that $\Omega_{R^{\prime}}^{1}(N / U N)$ is the kernel of the following matrix factorization of $f+U V$

$$
\sigma=\left(\left(\begin{array}{cc}
T-\phi & -U \\
V & \phi
\end{array}\right), \quad\left(\begin{array}{cc}
\phi & U \\
-V & T-\phi
\end{array}\right)\right)
$$

for a certain square $t$-matrix $\phi$ over $\Lambda[[T]]$ such that $(\phi, T-\phi) \in \operatorname{MF}_{\Lambda[[T]]}(f)$ and Coker $\phi \cong N / U N$. However, it seems hard to decompose $\sigma$ as it follows from $3.7 \mathrm{ii}$ ).

3.11 From now on let $\Lambda^{* *}:=\Lambda[[U, V]], R^{\prime}:=\Lambda^{* *} /(f+U V)$ as in Corollary 3.7 and $(\phi, \varphi)$ a matrix factorization of $f$ over $\Lambda$. Then

$$
F(\phi, \varphi):=\left(\left(\begin{array}{cc}
\phi & V \\
U & -\varphi
\end{array}\right), \quad\left(\begin{array}{cc}
\varphi & V \\
U & -\phi
\end{array}\right)\right)
$$

is a matrix factorization of $f+U V$ over $\Lambda^{* *}$. If $(\alpha, \beta):\left(\phi_{1}, \varphi_{1}\right) \rightarrow\left(\phi_{2}, \varphi_{2}\right)$ is a morphism in $\operatorname{MF}_{\Lambda}(f)$ then

$$
F(\alpha, \beta):=\left(\left(\begin{array}{cc}
\alpha & 0 \\
0 & \beta
\end{array}\right), \quad\left(\begin{array}{ll}
\beta & 0 \\
0 & \alpha
\end{array}\right)\right)
$$


is a morphism $F\left(\phi_{1}, \varphi_{1}\right) \rightarrow F\left(\phi_{2}, \varphi_{2}\right)$ in $\mathrm{MF}_{\Lambda} \cdots(f+U V) . \quad F$ defines an exact functor $\mathrm{MF}_{\Lambda}(f) \rightarrow \mathrm{MF}_{\Lambda} \cdots(f+U V)$ and the functor $H: \mathrm{MF}_{\Lambda} \cdots(f+U V) \rightarrow \mathrm{MF}_{\Lambda}(f)$ given by $U, V \rightarrow 0$ satisfies $H F \cong 1_{\mathrm{MF}_{(f)}} \oplus D$, where $D: \mathrm{MF}_{\Lambda}(f) \rightarrow \mathrm{MF}_{\Lambda}(f)$ is given by $(\phi, \varphi) \rightarrow(\varphi, \phi)$ (see [So] $(2.1))$. If $(\phi, \varphi)$ is a reduced matrix factorization then $F(\phi, \varphi)$ is also reduced. Similarly, $F(f, 1)$ and $F(1, f)$ are direct sums of $(f+U V, 1)$ and $(1, f+U V)$. Thus, $F$ induces an additive functor

$$
F: \underline{\operatorname{RMF}}_{\Lambda}(f) \rightarrow \underline{\operatorname{RMF}} \Lambda * *(f+U V) .
$$

3.12 Knörrer's Periodicity Theorem. $F$ induces an equivalence of the categories

$$
F: \underline{\operatorname{Lift}}(R, \bar{R}) \rightarrow \underline{\operatorname{Lift}}\left(R^{\prime}, \bar{R}\right) .
$$

In particular, $\operatorname{Lift}(R, \bar{R})$ and $\operatorname{Lift}\left(R^{\prime}, \bar{R}\right)$ are stable equivalent.

If char $K \neq 2$ the proof can be given as in [Kn] or [Yo] (12.9), (12.10) using Proposition 3.6 twice for $\lambda=0, \mu=1$ and Lemma 3.8. However, using Corollary 3.7 we can prove 3.12 independently of char $K$.

Lemma 3.13 Let $N \in \operatorname{Lift}\left(R^{\prime}, \bar{R}\right)$ be a non-free indecomposable module. Then there exists an indecomposable module $M \in \operatorname{Lift}(R, \bar{R})$ such that $N$ is a direct summand of $F(M)$.

Proof: By Corollary $3.7 N$ is a direct summand of $\Omega_{R^{\prime}}^{1}(N / U N)$ and $N / U N$ is a direct summand in $\Omega_{R[[T]]}^{1}(N /(U, V) N)$. Then $N$ is a direct summand of $\Omega_{R^{\prime}}^{1}\left(\Omega_{R[[T]]}^{1}(N /(U, V) N)\right)$. Let $G:=\Omega_{R^{\prime}}^{1}\left(\Omega_{R[T]]}^{1}(-)\right)$ be the composite functor $\operatorname{Lift}(R, \bar{R}) \rightarrow L\left(R^{\prime}, \bar{R}\right)$ and express $\widehat{N}:=$ $N /(U, T) N$ as a direct sum of indecomposable $R$-modules, say $\widehat{N}=\oplus_{i=1}^{s} P_{i}$. As $N$ is a direct summand of $G(\widehat{N})=\bigoplus_{i=1}^{s} G\left(P_{i}\right)$ we conclude that $N$ is a direct summand of a certain $G\left(P_{i}\right)$ ( $R^{\prime}$ is Henselian!).

Now we show that $G\left(P_{i}\right)$ and $F\left(P_{i}\right) \oplus F\left(\Omega_{R}^{1}\left(P_{i}\right)\right)$ are isomorphic modulo free $R$-modules. Let $(\phi, \varphi)$ be a reduced matrix factorization of $f$ over $\Lambda$ such that Coker $\phi \cong P_{i}$. According to Lemma 3.5 applied for $\lambda=\mu=0, T=U$ we see that $\widetilde{\Omega}_{R[[T]]}^{1}\left(P_{i}\right)$ is the cokernel of the reduced matrix factorization

$$
\left(\left(\begin{array}{rr}
\varphi & -T \\
0 & \phi
\end{array}\right), \quad\left(\begin{array}{ll}
\phi & T \\
0 & \varphi
\end{array}\right)\right)
$$

of $f$ over $\Lambda[[T]]$. Applying again Lemma 3.5 for $\lambda=T, \mu=1$ we see that $G\left(P_{i}\right)$ is the cokernel of the following reduced matrix factorization of $f+U V$ over $\Lambda^{* *}$ :

$$
\left.\left(\begin{array}{rr|rr}
\phi & T & -U & 0 \\
0 & \varphi & 0 & -U \\
\hline V & 0 & \varphi & -T \\
0 & V & 0 & \phi
\end{array}\right), \quad\left(\begin{array}{rr|rr}
\varphi & -T & U & 0 \\
0 & \phi & 0 & U \\
\hline-V & 0 & \phi & T \\
0 & -V & 0 & \varphi
\end{array}\right)\right) .
$$

Let $\sigma$ be the first matrix above. By elementary transformations we note that

$$
\sigma \sim\left(\begin{array}{rr|rr}
\phi & -V & -U & 0 \\
U & \varphi & 0 & -U \\
\hline 0 & 0 & \varphi & V \\
0 & 0 & -U & \phi
\end{array}\right) \sim\left(\begin{array}{rr|rr}
\phi & -V & 0 & 0 \\
U & \varphi & 0 & 0 \\
\hline 0 & 0 & \varphi & V \\
0 & 0 & -U & \phi
\end{array}\right)
$$


(add column 3 to column 2, then subtract row 2 from row 3, then add row 1 to row 4 , then subtract column 4 from column $1, \ldots)$. Hence, $G\left(P_{i}\right) \cong F\left(P_{i}\right) \oplus F\left(\Omega_{R}^{1}\left(P_{i}\right)\right)$. As $N$ is indecomposable it should be a direct summand of $F(M)$ for $M:=P_{i}$ or $\Omega_{R}^{1}\left(P_{i}\right)\left(R^{\prime}\right.$ is Henselian!). Due to $2.7 \Omega_{R}^{1}\left(P_{i}\right)$ is indecomposable.

Lemma 3.14 $F$ is fully faithful.

Proof: We follow [Yo] (12.10) (see also [So] (3.1)). Let $(\phi, \varphi),\left(\phi^{\prime}, \varphi^{\prime}\right) \in \underline{\mathrm{RMF}}_{\Lambda}(f)$ and $E=$ Coker $\phi, E^{\prime}=$ Coker $\phi^{\prime}$. We have to show that $F$ induces a bijection

$$
\rho: \underline{\operatorname{Hom}}_{R}\left(E, E^{\prime}\right) \rightarrow \underline{\operatorname{Hom}}_{R^{\prime}}\left(F(E), F\left(E^{\prime}\right)\right),
$$

where $\underline{\operatorname{Hom}}_{R}$ defines the usual Hom for the category $\underline{\operatorname{Mod}} R$.

Let $\Omega_{R}^{i}(E)$ be the $i$-th syzygy of $E$ over $R, i \geq 1$. Since the minimal free resolution of $E$ has periodicity 2 (see 2.4 ) we obtain $\Omega_{R}^{2}(E) \cong E$. Thus we have an exact sequence

$$
0 \rightarrow E \cong \Omega_{R}^{2}(E) \stackrel{j}{\rightarrow} R^{s} \rightarrow \Omega_{R}^{1}(E) \rightarrow 0
$$

for an $s \in \mathbb{N}$. Put $\widehat{E}:=\Omega_{R}^{1}(E)$. Applying $\operatorname{Hom}_{R}\left(-, E^{\prime}\right)$ and $\operatorname{Hom}_{R^{\prime}}\left(F(-), F\left(E^{\prime}\right)\right)$ to (12) we obtain the following commutative diagram

$$
\begin{array}{ccccc}
\operatorname{Hom}_{R}\left(R^{s}, E^{\prime}\right) & \stackrel{j^{*}}{\rightarrow} & \operatorname{Hom}_{R}\left(E, E^{\prime}\right) & \stackrel{\mu}{\rightarrow} & \operatorname{Ext}{ }_{R}^{1}\left(\widehat{E}, E^{\prime}\right) \\
\operatorname{Hom}_{R^{\prime}}\left(F(R)^{s}, F\left(E^{\prime}\right)\right) & \rightarrow & \operatorname{Hom}_{R^{\prime}}\left(F(E), F\left(E^{\prime}\right)\right) & \stackrel{\nu}{\rightarrow} & \operatorname{Ext}_{R^{\prime}}^{1}\left(F(\widehat{E}), F\left(E^{\prime}\right)\right)
\end{array}
$$

where the rows are exact, $\mu, \nu$ are surjective $(F(R)$ is free!) and the vertical maps are induced by the exact functor $F$ (see 3.11). Note that $\mu, \nu$ induce the surjections $\operatorname{Ext}_{R}^{1}\left(\widehat{E}, E^{\prime}\right) \stackrel{\gamma}{\rightarrow}$ $\underline{\operatorname{Hom}}_{R}\left(E, E^{\prime}\right), \operatorname{Ext}_{R^{\prime}}^{1}\left(F(\widehat{E}), F\left(E^{\prime}\right)\right) \stackrel{\tau}{\rightarrow} \underline{\operatorname{Hom}}_{R^{\prime}}\left(F(E), F\left(E^{\prime}\right)\right)$. It is enough to show that the last vertical map $\theta$ from the diagram is bijective. Indeed, then we obtain $\sigma$ surjective from the following commutative diagram

$$
\begin{array}{ccc}
\operatorname{Ext}_{R}^{1}\left(\widehat{E}, E^{\prime}\right) & \stackrel{\theta}{\rightarrow} & \operatorname{Ext}_{R^{\prime}}^{1}\left(F(\widehat{E}), F\left(E^{\prime}\right)\right) \\
\downarrow & & \downarrow \\
\underline{\operatorname{Hom}}_{R}\left(E, E^{\prime}\right) & \stackrel{\rho}{\rightarrow} & \underline{\operatorname{Hom}}_{R^{\prime}}\left(F(E), F\left(E^{\prime}\right)\right)
\end{array}
$$

because the vertical maps $\gamma, \tau$ are surjective. If $\rho(\delta)=0$ for $\delta \in \underline{\operatorname{Hom}}_{R}\left(E, E^{\prime}\right)$ then $F(\delta)$ factorizes by a free $R^{\prime}$-module and thus $\delta \oplus D(\delta) \cong H F(\delta)$ (and so $\delta$ ) factorizes by a free $R$-module as well (see 3.11). Hence, $\delta=0$ in $\underline{\operatorname{Hom}}_{R}\left(E, E^{\prime}\right)$.

It remains to show that $\theta$ is a bijection. Let $(\alpha, \beta):(\phi, \varphi) \rightarrow\left(\phi^{\prime}, \varphi^{\prime}\right)$ be a morphism in $\underline{\operatorname{RMF}}_{\Lambda}(f)$ corresponding to a certain $h \in \underline{\operatorname{Hom}}_{R}\left(E, E^{\prime}\right), h:=\operatorname{Coker}(\alpha, \beta)$. Then $\mu(h)$ corresponds to the extension

$$
\left(\begin{array}{ll}
\phi^{\prime} & \alpha \\
0 & \varphi
\end{array}\right)
$$

and similarly $\nu F(h)$ corresponds to the extension

$$
\left(\begin{array}{cc|cc}
\phi^{\prime} & V & \alpha & 0 \\
U & -\varphi^{\prime} & 0 & \beta \\
\hline 0 & 0 & \varphi & V \\
0 & 0 & U & -\phi
\end{array}\right) .
$$

Now the proof can be given as in [Yo] (12.10). 
Remark 3.15 In fact the statement of Lemma 3.14 is weaker than we really prove there. The bijectivity of $\theta$ means, in fact, that the factor of $\operatorname{Hom}_{R}\left(E, E^{\prime}\right)$ by the submodule (maps factorizing by $j\rangle$ is isomorphic with the factor of $\operatorname{Hom}_{R^{\prime}}\left(F(E), F\left(E^{\prime}\right)\right)$ by the submodule $\langle$ maps factorizing by $F(j)\rangle$.

3.16 Proof of Theorem 3.12. It remains to show that $F$ gives a surjective map onto the set of objects of $\underline{\operatorname{Lift}}\left(R^{\prime}, \bar{R}\right)$. Let $N \in \operatorname{Lift}\left(R^{\prime}, \bar{R}\right)$ be a non-free indecomposable module. According to Lemma 2.13, $N$ is a direct summand of $F(M)$ for a certain indecomposable module $M \in \operatorname{Lift}(R, \bar{R})$. As $F$ is fully faithful by Lemma 3.14 we see that $F(M)$ is indecomposable in $\underline{\operatorname{Lift}}\left(R^{\prime}, \bar{R}\right)$ and so in $\operatorname{Lift}\left(R^{\prime}, \bar{R}\right)$, too. Hence, $N \cong F(M)$.

Theorem 3.17 Let $(B, \wp, K)$ be a Noetherian, Henselian local ring, $\Lambda:=B[[X]], X=$ $\left(X_{1}, \ldots, X_{r}\right), f \in \Lambda$ a non-unit formal power series which is not in $\wp \Lambda, \mathcal{R}:=\Lambda /(f), \mathcal{R}^{\prime}:=$ $\Lambda[[U, V]] /(f+U V), R:=K \otimes_{B} \mathcal{R}, R^{\prime}:=K \otimes_{B} \mathcal{R}^{\prime}$ and $\mathcal{D}_{B}^{R}$ (resp. $\left.\mathcal{D}_{B}^{R^{\prime}}\right)$ the category of all finitely generated $\mathcal{R}$-modules $E$ (resp. $\mathcal{R}^{\prime}$-modules $E^{\prime}$ ) which are flat over $B$ and such that $K \otimes_{B} E \in M C M(R)$ (resp. $K \otimes_{B} E^{\prime} \in M C M\left(R^{\prime}\right)$ ). Suppose $\mathcal{R}$ and $\mathcal{R}^{\prime}$ are flat over $B$. Then there exists an equivalence

$$
F: \mathcal{D}_{B}^{R} /\{\mathcal{R}\} \rightarrow \mathcal{D}_{B}^{R^{\prime}} /\left\{\mathcal{R}^{\prime}\right\}
$$

In particular, $\mathcal{D}_{B}^{R}, \mathcal{D}_{B}^{R^{\prime}}$ are stable equivalent.

For the proof apply Theorem 3.12 in the frame of Theorem 2.8 .

Corollary 3.18 With the hypothesis and notation from Theorem 3.17, $F$ yields a bijection preserving the indecomposability from the set of isomorphism classes of modules from $\mathcal{D}_{B}^{R}$ which have no free summands onto the set of isomorphism classes of modules from $\mathcal{D}_{B}^{R^{\prime}}$ which have no free summands.

Corollary 3.19 With the hypothesis and notation from Theorem 3.17, let $M$ be a maximal Cohen-Macaulay $R$-module without free summands, $B:=K[\varepsilon], \varepsilon^{2}=0$ and $\mathcal{R}:=B \otimes_{K} R=$ $R[\varepsilon]$. Then $F$ induces a bijection $\operatorname{Def}_{M}(K[\varepsilon]) \rightarrow \operatorname{Def}_{F(M)}(K[\varepsilon])$.

Proof: There exists a canonical bijection $\operatorname{Def}_{M}(K[\varepsilon]) \rightarrow \operatorname{Ext}_{R}^{1}(M, M)$. Thus it is enough to see that $F$ induces a bijection

$$
\operatorname{Ext}_{R}^{1}(M, M) \rightarrow \operatorname{Ext}_{R^{\prime}}^{1}(F(M), F(M)) .
$$

But this was done in the proof of Lemma 3.14.

Theorem 3.20 With the hypothesis and notation from Theorem 3.17, let $M$ be a non-free indecomposable maximal Cohen-Macaulay $R$-module and $D e f_{M}^{R}(B)$ the set of isomorphism classes of deformations of $M$ over $B$ (see 2.13). Then $F$ induces a bijection

$$
\omega_{B}: \operatorname{Def}_{M}^{\mathcal{R}}(B) \rightarrow \operatorname{Def}_{F(M)}^{\mathcal{R}^{\prime}}(B)
$$


Proof: We denoted by $F$ both functors $\mathcal{D}_{B}^{R} /\{\mathcal{R}\} \rightarrow \mathcal{D}_{B}^{R^{\prime}} /\left\{\mathcal{R}^{\prime}\right\}, \operatorname{MCM}(R) /\{R\} \rightarrow$ $\operatorname{MCM}\left(R^{\prime}\right) /\left\{R^{\prime}\right\}$. Let $(E, \xi),\left(E^{\prime}, \xi^{\prime}\right)$ be two deformations of $F(M)$ over $B$. If $(E, \xi) \cong$ $\left(E^{\prime}, \xi^{\prime}\right)$, then, clearly, $(F(E), F(\xi)) \cong\left(F\left(E^{\prime}\right), F\left(\xi^{\prime}\right)\right)$, that is $\omega_{B}$ is well-defined. Conversely, if $(F(E), F(\xi)) \cong\left(F\left(E^{\prime}\right), F\left(\xi^{\prime}\right)\right)$, then there exists an $R^{\prime}$-isomorphism $\alpha: F(E) \rightarrow F\left(E^{\prime}\right)$ such that $(K \otimes \alpha) F(\xi)=F\left(\xi^{\prime}\right)$. We have a commutative diagram as in the proof of Lemma 3.14 (see also 3.15 )

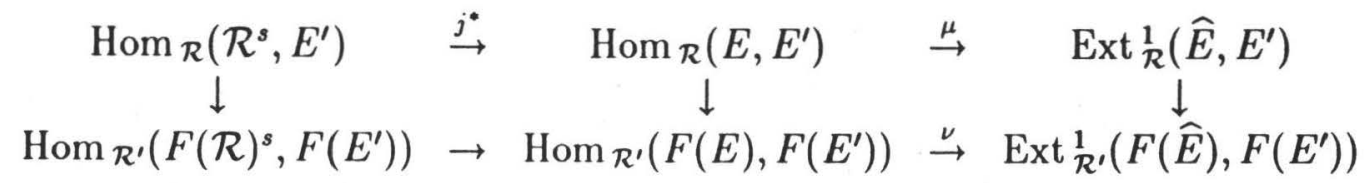

where $\widehat{E}:=\Omega_{\mathcal{R}}^{1}(E)$, the rows are exact, $\mu, \nu$ are surjective $(F(\mathcal{R})$ is free!), the last vertical map $\theta$ is bijective and $j^{*}$ is induced by $j$ from the following exact sequence

$$
0 \rightarrow E \stackrel{j}{\rightarrow} \mathcal{R}^{s} \rightarrow \widehat{E} \rightarrow 0 .
$$

Then there exists $\beta: E \rightarrow E^{\prime}$ such that $\theta \mu(\beta)=\nu(\alpha)$, that is $h:=F(\beta)-\alpha$ factorizes by $F(j)$. Tensorizing (13) by $K \otimes_{B}$ - we obtain the following exact sequence

$$
0 \rightarrow K \otimes_{B} E \stackrel{\bar{J}}{\rightarrow} R^{s} \rightarrow \Omega_{R}^{1}\left(K \otimes_{B} E\right) \cong K \otimes_{B} \widehat{E} \rightarrow 0 .
$$

Thus $K \otimes_{B} h$ factorizes by $F(\bar{\jmath})$. Put $\bar{\lambda}:=K \otimes_{B} \beta-\xi^{\prime} \xi^{-1}$. Then $F(\bar{\lambda})=F\left(K \otimes_{B} \beta\right)$ $-F\left(\xi^{\prime}\right) F(\xi)^{-1}=K \otimes_{B}(F(\beta)-\alpha)=K \otimes_{B} h$. Passing from $\left(B, \mathcal{R}, \mathcal{R}^{\prime}, E, E^{\prime}, \widehat{E}\right)$ to $\left(K, R, R^{\prime}, \bar{E}:=K \otimes_{B} E, \bar{E}^{\prime}:=K \otimes_{B} E^{\prime}, \widehat{E}:=K \otimes_{B} \widehat{E}\right)$ the above commutative diagram becomes

$$
\begin{array}{ccccc}
\operatorname{Hom}_{R}\left(R^{s}, \bar{E}^{\prime}\right) & \stackrel{\vec{\jmath}}{\rightarrow} & \operatorname{Hom}_{R}\left(\bar{E}, \bar{E}^{\prime}\right) & \stackrel{\bar{\mu}}{\rightarrow} & \operatorname{Ext}_{R}^{1}\left(\overline{\widehat{E}}, \bar{E}^{\prime}\right) \\
\downarrow & & \downarrow & & \downarrow \\
\operatorname{Hom}_{R^{\prime}}\left(F(R)^{s}, F\left(\bar{E}^{\prime}\right)\right) & \rightarrow & \operatorname{Hom}_{R^{\prime}}\left(F(\bar{E}), F\left(\bar{E}^{\prime}\right)\right) & \stackrel{\bar{\nu}}{\rightarrow} & \operatorname{Ext}_{R^{\prime}}^{1}\left(F(\overline{\widehat{E}}), F\left(\bar{E}^{\prime}\right)\right)
\end{array}
$$

where the rows are exact, $\bar{\mu}, \bar{\nu}$ are surjective, the last vertical map $\bar{\theta}$ is bijective and $\bar{j}^{*}$ is induced by $\bar{\jmath}$. As $F(\bar{\lambda})=K \otimes_{B} h$ factorizes by $\bar{\jmath}$ we have $\bar{\theta} \bar{\mu}(\bar{\lambda})=\bar{\nu}(F(\bar{\lambda}))=0$. Thus, $\bar{\mu}(\bar{\lambda})=0, \bar{\theta}$ being injective and so $\bar{\lambda}$ factorizes by $\bar{\jmath}$, let us say $\bar{\lambda}=\bar{k} \bar{\jmath}$ for an $R$-morphism $\bar{k}: R^{s} \rightarrow \bar{E}^{\prime}$. Since $R^{s}$ is free we may lift $\bar{k}$ to a map $k: \mathcal{R}^{s} \rightarrow E^{\prime}$. Then $\lambda:=k j$ lifts $\bar{\lambda}$ and we see that $\beta^{\prime}:=\beta-\lambda$ satisfies $\left(K \otimes_{B} \beta^{\prime}\right) \xi=\left(K \otimes_{B} \beta\right) \xi-\left(K \otimes_{B} \lambda\right) \xi=\left(K \otimes_{B} \beta\right) \xi-\bar{\lambda} \xi=\xi^{\prime}$. But $\operatorname{End}_{R} M$ is local because $M$ is indecomposable and $R$ is Henselian. Then $\beta^{\prime}$ is an isomorphism since $K \otimes_{B} \beta^{\prime}=\xi^{\prime} \xi^{-1}$ is so. Hence, $(E, \xi) \cong\left(E^{\prime}, \xi^{\prime}\right)$, that is $\omega_{B}$ is injective.

It remains to prove the surjectivity of $\omega_{B}$. By Theorem 3.17 any deformation of $F(M)$ over $B$ has the form $(F(E), \sigma)$ for an $E \in \mathcal{D}_{B}^{R}$ and an $R$-isomorphism $\sigma: F(M) \rightarrow K \otimes_{B} F(E) \cong F(\bar{E})$. As $\bar{\theta}$ is surjective there exists an $R$-morphism $\chi: M \rightarrow \bar{E}$ such that $\bar{g}:=\sigma-F(\chi)$ factorizes by $F(\bar{\jmath})$. Since $F(M)$ is indecomposable $\operatorname{End}_{R^{\prime}}(F(M))$ is local and $F(\chi)$ must be an $R^{\prime}$-isomorphism. Then $\chi$ is an $R$-isomorphism, too, because $F$ defines an equivalence $\mathcal{D}_{B}^{R} /\{\mathcal{R}\} \rightarrow \mathcal{D}_{B}^{R^{\prime}} /\left\{\mathcal{R}^{\prime}\right\}$ and $\operatorname{End}_{R}(M)$ is local. Now $\bar{g}$ can be lifted to an $\mathcal{R}$-endomorphism $g$ of $F(E)$ factorizing $F(j)$ (as $\bar{\lambda}$ above!). Then $F(\chi)=\sigma-(K \otimes g)=(K \otimes \eta) \sigma$ for $\eta:=1-g \sigma^{-1}$. Again $\eta$ is an isomorphism because $g$ factorizes $F(j)$ and $\operatorname{End}_{\mathcal{R}} F(E)$ is local. Hence, $(F(E), \sigma) \cong(F(E), F(\chi))$, that is $\omega_{B}$ is surjective. 
Theorem 3.21 With the hypothesis and notation from Theorem 3.17, let $S:=$ $\Lambda /\left(X_{1}^{2}, \ldots, X_{r-1}^{2}\right), \mathcal{H}:=S /(f), H:=K \otimes_{B} \mathcal{H}$ and $\mathcal{L}_{B}^{H}$ be the category of all finitely generated $\mathcal{H}$-modules $E$ which are flat over $B$ and such that $\bar{E}:=K \otimes_{B} E$ satisfies

$$
\left(\left(X_{1}, \ldots, X_{j-1}\right) \bar{E}: X_{j}\right)_{E}=\left(X_{1}, \ldots, X_{j}\right) \bar{E}
$$

for every $j, 1 \leq j<r$. Let $\mathcal{H}^{\prime}:=S[[U, V]] /(f+U V), H^{\prime}:=K \otimes_{B} \mathcal{H}^{\prime}$ and $\widehat{\mathcal{L}}_{B}^{H^{\prime}}$ be the category of all finitely generated $\mathcal{H}^{\prime}$-modules $E^{\prime}$ which are flat over $B$ and such that $\bar{E}^{\prime}:=K \otimes_{B} E^{\prime}$ satisfies $(\mathcal{L})$ and the system $\{U, V\}$ is regular on $\bar{E}^{\prime} /\left(X_{1}, \ldots, X_{r-1}\right) \bar{E}^{\prime}$. Suppose that $\mathcal{H}$ and $\mathcal{H}^{\prime}$ are flat $B$-algebras. Then the categories $\mathcal{L}_{B}^{H}$ and $\widehat{\mathcal{L}}_{B}^{H^{\prime}}$ are stable equivalent.

For the proof take $a=\left(\wp, X_{1}, \ldots, X_{r-1}\right)$ and note that $\mathcal{L}_{B}^{H}=\operatorname{Lift}(\mathcal{H}, \mathcal{H} / a \mathcal{H})$ as in theorem 2.11 and $\operatorname{Lift}\left(\mathcal{H}^{\prime}, \mathcal{H} / a \mathcal{H}\right)=\widehat{\mathcal{L}}_{B}^{H^{\prime}}$ since $\mathcal{H} / a \mathcal{H} \cong \mathcal{H}^{\prime} /(a, U, V) \mathcal{H}^{\prime}$ and $\{U, V\}$ is a regular system on $\mathcal{H}^{\prime} / a \mathcal{H}^{\prime}$. Then apply theorem 3.12 . 


\section{References}

[ADS] Auslander, M.; Ding, S.; Solberg, Ø: Liftings and Weak Liftings of Modules. J. of Algebra 156, 273-317, 1993.

[BGS] Buchweitz, R.-O.; Greuel, G.-M.; Schreyer, F.-O.: Cohen-Macaulay modules on hypersurface singularities II. Invent. Math. 88, 165-182, 1987.

[BH] Bruns, W.; Herzog, J.: Cohen-Macaulay-Rings. Cambridge University Press, 1993.

[CHP] Cipu, M.; Herzog, J.; Popescu, D.: Indecomposable generalized Cohen-Macaulay modules. Trans AMS, 342, 107-136, 1994.

[Di] Dieterich, E.: Reduction of isolated singularities. Comment. Math. Helvetici, 62, 654-676, 1987.

[DS] Ding, S.; Solberg, Ø: The Maranda Theorem and Liftings of Modules. Communications in Alg., 21, 1161-1187, 1993.

[Ei] Eisenbud, D.: Homological algebra on a complete intersection with an application to group representations. Trans AMS, 260,1, 35-64, 1980.

[GP] Greuel, G.-M.; Pfister, G.: Moduli spaces for torsion free modules on curve singularities I. J. of Algebraic Geometry, 2, 81-135, 1993.

[GK] Greuel, G.-M.; Knörrer, H.: Einfache Kurvensingularitäten und torsionsfreie Moduln. Math. Ann., 270, 417-425, 1985.

[GKr] Greuel, G.-M.; Kröning, H.: Simple singularities in positive characteristic. Math. Z. 203, 339-354, 1990.

[Kn] Knörrer, H.: Cohen-Macaulay modules on hypersurface singularities I. Invent. Math., 88, 153-164, 1987.

[Ma] Matsumura, H.: Commutative Algebra. Benjamin, 2nd Edition. Mass., 1980.

[PS] Pfister, G.; Steenbrink, J.H.M.: Reduced Hilbert schemes for irreducible curve singularities. J. Pure and Appl. Alg., 77, 103-116, 1992.

[Pi] Pierce, R.S.: Associative algebras. Graduate Texts in Math., 88, Springer-Verlag, 1982.

[Po1] Popescu, D.: Indecomposable Cohen-Macaulay modules and their multiplicities. Trans. AMS, 323, 369-387, 1991.

[Po2] Popescu, D.: Maximal Cohen-Macaulay modules over isolated singularities. Preprint Kaiserslautern, 1994.

[PR] Popescu, D.; Roczen, M.: Indecomposable Cohen-Macaulay modules and irreducible maps. Compositio Math., 76, 277-294, 1990. 
[Sc] Schreyer, F.-O.: Finite and countable CM-representation type. In Singularities, Representation of algebras and vector bundles. Springer Lect. Notes in Math., 1273, 9-34, 1987.

[So] Solbert, Ø: Hypersurface singularities of finite Cohen-Macaulay type. Proc. London Math. Soc., (3), 58, 258-280, 1989.

[Yo] Yoshino, Y.: Cohen-Macaulay Modules over Cohen-Macaulay Rings. London Math. Soc. Lect. Note Ser., 146, Cambridge, 1990. 